\title{
A surge of light at the birth of a supernova
}

\author{
M. C. Bersten ${ }^{1,2,3}$, G. Folatelli1 ${ }^{1,2,3}$, F. García ${ }^{2,4,5}$, S. D. Van Dyk ${ }^{6}$, O. G. Benvenuto ${ }^{1,2}$, M. Orellana ${ }^{7}$, V. Buso ${ }^{8}$, J. L. Sánchez ${ }^{9}$, \\ M. Tanaka ${ }^{10}$, K. Maeda ${ }^{3,11}$, A. V. Filippenko ${ }^{12,13}$, W. Zheng ${ }^{12}$, T. G. Brink ${ }^{12}$, S. B. Cenko ${ }^{14,15}$, T. de Jaeger ${ }^{12}$, S. Kumar ${ }^{16}$, T. J. Moriya ${ }^{10}$, \\ K. Nomoto ${ }^{3}$, D. A. Perley ${ }^{17}$, I. Shivvers ${ }^{12} \&$ N. Smith ${ }^{18}$
}

\begin{abstract}
It is difficult to establish the properties of massive stars that explode as supernova $\mathrm{e}^{1,2}$. The electromagnetic emission during the first minutes to hours after the emergence of the shock from the stellar surface conveys important information about the final evolution and structure of the exploding star ${ }^{3-6}$. However, the unpredictable nature of supernova events hinders the detection of this brief initial phase $^{7-9}$. Here we report the serendipitous discovery of a newly born, normal type IIb supernova $(\mathrm{SN} 2016 \mathrm{gkg})^{10}$, which reveals a rapid brightening at optical wavelengths of about 40 magnitudes per day. The very frequent sampling of the observations allowed us to study in detail the outermost structure of the progenitor of the supernova and the physics of the emergence of the shock. We develop hydrodynamical models of the explosion that naturally account for the complete evolution of the supernova over distinct phases regulated by different physical processes. This result suggests that it is appropriate to decouple the treatment of the shock propagation from the unknown mechanism that triggers the explosion.
\end{abstract}

On 2016 September 20 (dates are given in UT throughout), amateur astronomer V.B. was testing a camera mounted on his $40-\mathrm{cm}$ Newtonian telescope. He pointed the telescope at NGC 613, a spiral galaxy at a distance of $26.4 \mathrm{Mpc}$, because at that time it was located near the zenith. Over approximately $1.5 \mathrm{~h}$ he imaged the galaxy with a clear filter while a supernova was being born, using 20-s exposures to avoid saturation caused by the bright city sky. An initial series of 40 images obtained during $20 \mathrm{~min}$ showed no sign of the supernova. From the combined image we obtained a $5 \sigma$ detection limit of 19.4 mag converted to the $V$ band (see Methods). When observations resumed, after an interval of $45 \mathrm{~min}$, the supernova became visible. During the remaining 25 min of observations the supernova doubled its flux (Fig. 1, Methods). The deep detection limit and frequent sampling during the initial rise constitute an unprecedented set of observations for a supernova discovery. A linear fit to the discovery photometry yields a remarkably fast rise rate of $43 \pm 6 \mathrm{mag} \mathrm{d}^{-1}$. An extrapolation of this linear rise back in time to the proposed brightness of the progenitor of $V \approx 24$ mag (Methods) suggests that SN 2016gkg exploded some time between 2:50 UT and 5:35 UT. This constraint of less than $3 \mathrm{~h}$ on the explosion epoch is one of the most stringent available $e^{11-15}$.

The prompt discovery and announcement of SN $2016 \mathrm{gkg}^{10}$ triggered extensive monitoring that began less than one day later, including Swift $\mathrm{X}$-ray, ultraviolet and optical observations ${ }^{16,17}$. This permitted excellent coverage of the subsequent evolution of the supernova. Consequently, the cooling peak, which lasted for about three days, is one of the best observed so far. Follow-up spectroscopy provided a classification of
SN $2016 \mathrm{gkg}$ as a type IIb supernova ${ }^{16}$. Our photometric and spectroscopic monitoring, which began less than one day after discovery, is described in Methods. In addition to these observations, the Hubble Space Telescope (HST) archive contained images of the site of the supernova, obtained with the WFPC2 camera in 2001. An object was identified at the location of the supernova in all three available optical bands (F450W, F606W and F814W). Our analysis of these data and inferences about the progenitor system are given in Methods ${ }^{16,18}$.

To interpret the physical process that governed the behaviour of the supernova at the time of discovery, we plot the luminosity versus the rise rate and compare these data with available early optical observations of other supernovae and transients (Fig. 2; see ref. 9 for a similar

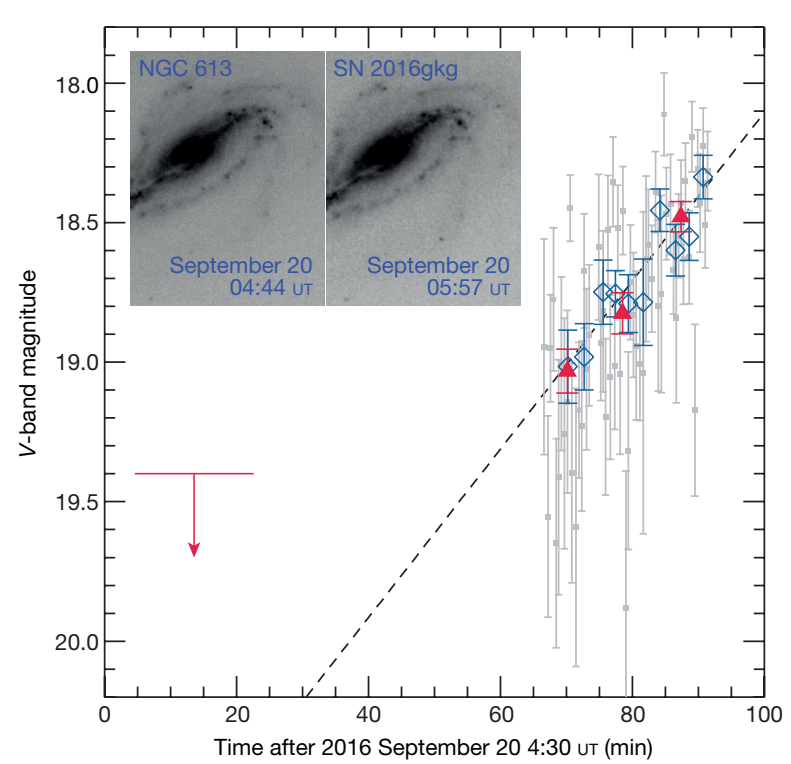

Figure 1 Photometry of SN 2016gkg at discovery. The data show a $5 \sigma$ detection limit (red arrow) and a sharp rise (points) starting less than $1 \mathrm{~h}$ after discovery. The inset images display a combination of the first series of 40 images (left) and a combination of the last series of 21 images (right). Photometry is shown for individual images (grey squares), combinations of 5 or 6 images (blue diamonds) and combinations of 17-21 images (red triangles). The dashed line is a linear fit to the blue diamonds, with a slope of $43 \pm 6 \mathrm{mag} \mathrm{d}^{-1}$. Error bars are $1 \sigma$. Photometry from combined images reveals hints of structure around the linear fit, although its statistical significance is low (the reduced $\chi^{2}$ of the linear fit is 0.85 ).

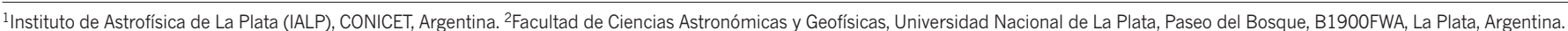
${ }^{3}$ Kavli Institute for the Physics and Mathematics of the Universe, Todai Institutes for Advanced Study, University of Tokyo, 5-1-5 Kashiwanoha, Kashiwa, Chiba 277-8583, Japan. ${ }^{4}$ Instituto Argentino

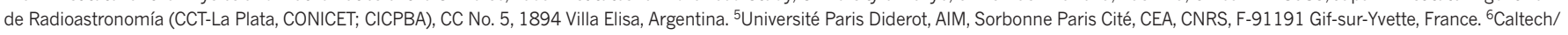

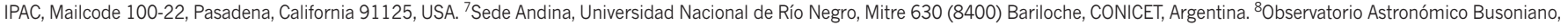
Entre Ríos 2974 (2000), Rosario, Argentina. ${ }^{9}$ Observatorio Astronómico Geminis Austral, Rosario, Argentina. ${ }^{10}$ Division of Theoretical Astronomy, National Astronomical Observatory of Japan, National Institutes of Natural Sciences, 2-21-1 Osawa, Mitaka, Tokyo 181-8588, Japan. ${ }^{11}$ Department of Astronomy, Kyoto University, Kitashirakawa-Oiwake-cho, Sakyo-ku, Kyoto 606-8502, Japan. ${ }^{12}$ Department of Astronomy, University of California, Berkeley, California 94720-3411, USA. ${ }^{13}$ Miller Senior Fellow, Miller Institute for Basic Research in Science, University of California, Berkeley, California 94720, USA. ${ }^{14}$ Astrophysics Science Division, NASA Goddard Space Flight Center, Greenbelt, Maryland 20771, USA. ${ }^{15}$ Joint Space-Science Institute, University of Maryland, College Park, Maryland 20742, USA. ${ }^{16}$ Department of Physics, Florida State University, 77 Chieftain Way, Tallahassee, Florida 32306, USA. ${ }^{17}$ Astrophysics Research Institute, Liverpool John Moores University, IC2, Liverpool Science Park, 146 Brownlow Hill, Liverpool L3 5RF, UK. ${ }^{18}$ Steward Observatory, University of Arizona, 933 North Cherry Avenue, Tucson, Arizona 85721, USA.
} 


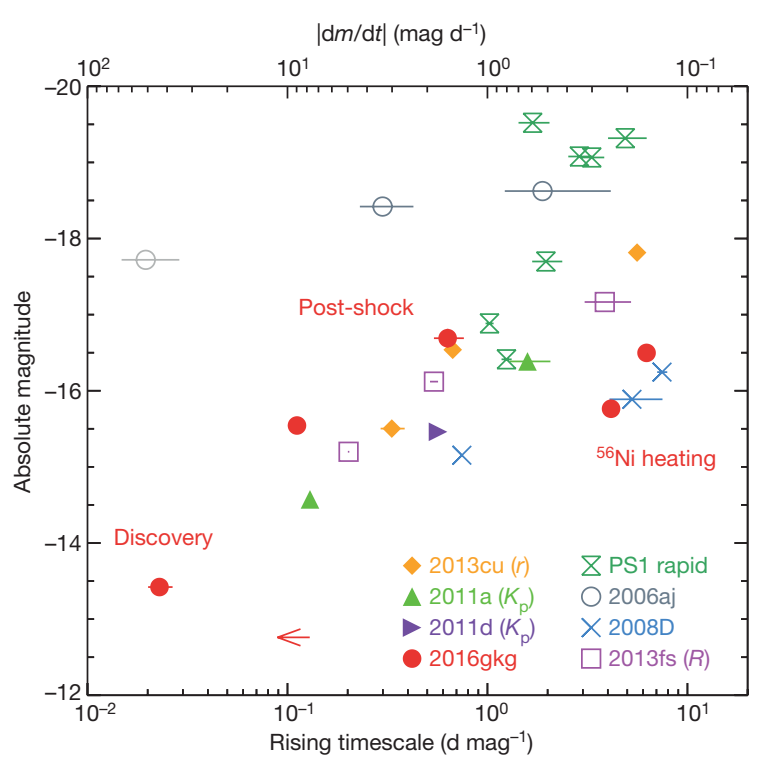

Figure 2 | Luminosity versus rise rate for objects with early optical detections. The rate of change of magnitude per unit time, $\mathrm{d} m / \mathrm{d} t$, is shown on the upper $x$ axis. $V$-band data are used, except as indicated in parentheses next to the name of the object. SN $2016 \mathrm{gkg}$ is shown with filled red circles following its light-curve evolution, and an upper limit (red arrow) from the pre-discovery detection limit. The point labelled 'discovery' represents the initial rise. 'PS1 rapid' indicates rapidly evolving transients from Pan-STARRS1 ${ }^{27}$. Each point is one of the following objects (in parentheses we give the observed band that best matches the $V$ band, according to the redshift of each object): PS1-10ah (rP1), PS1-10bjp (rP1), PS1-11qr (iP1), PS1-11bbq (zP1), PS1-12bv (iP1), PS1-12brf (iP1) and PS1-13duy (iP1). Other supernovae are as follows: the broad-lined type Ic, GRB-associated supernova SN 2006aj (dark-grey circles ${ }^{28}$ and light-grey circle $^{11}$, but see a discussion about the light-grey data point in Methods); the X-ray flash associated with the type Ib supernova SN 2008D ${ }^{12}$; the type II supernova SN 2013fs (iPTF13dqy) ${ }^{15}$; the type IIb supernova SN $2013 \mathrm{cu}$ (iPTF13ast) ${ }^{13}$; and the type II supernovae from the Kepler mission, KSN 2011a and KSN 2011 ${ }^{14}$. Error bars are $1 \sigma$.

graph in the near-ultraviolet range). The low luminosity and fast rise seen in SN $2016 \mathrm{gkg}$ place the discovery data in a clearly different location on the diagram compared with later observations and with data for other objects. This suggests a different physical origin for the initial rise of SN $2016 \mathrm{gkg}$.

A natural explanation for the above comparison is that the first signal of SN $2016 \mathrm{gkg}$ corresponds to the long-sought shock-breakout phase $^{19-21}$. Hydrodynamical simulations indicate that, although the shock-breakout signal is predominant in the X-ray and ultraviolet ranges, it has a clear manifestation in the optical range as well, characterized by an extremely rapid brightening at relatively low luminosity ${ }^{6,22}$. To test this interpretation, we performed numerical modelling of the supernova observations.

We divided the supernova modelling into two main stages ${ }^{23}$. First, the overall evolution of the supernova was modelled by adjusting the explosion energy, ejecta mass and ${ }^{56} \mathrm{Ni}$ mass to reproduce the main light-curve peak and expansion velocities from spectral lines. In this way, we found that a model with an energy of $E \approx 1.2 \times 10^{51} \mathrm{erg}$, an ejecta mass of $M_{\mathrm{ej}} \approx 3.4 M_{\odot}$ (where $M_{\odot}$ is the mass of the Sun) and a ${ }^{56} \mathrm{Ni}$ mass of $M_{\mathrm{Ni}} \approx 0.085 M_{\odot}$ provided a good match to the observations (see Methods for alternative solutions). Once these parameters were constrained, the second step consisted of modelling the postshock cooling peak. It is well known that to reproduce such a peak, an extended $\mathrm{H}$-rich envelope has to be attached to the usual progenitor structure from stellar-evolution calculations. We varied the radius and mass of the envelope and arrived at a radius of $R_{\mathrm{env}} \approx 320 R_{\odot}$ (where $R_{\odot}$ is the radius of the Sun) and a mass of $M_{\text {env }} \approx 0.01 M_{\odot}$ for the extended envelope (see refs 16 and 17 for other estimates). Our preferred model

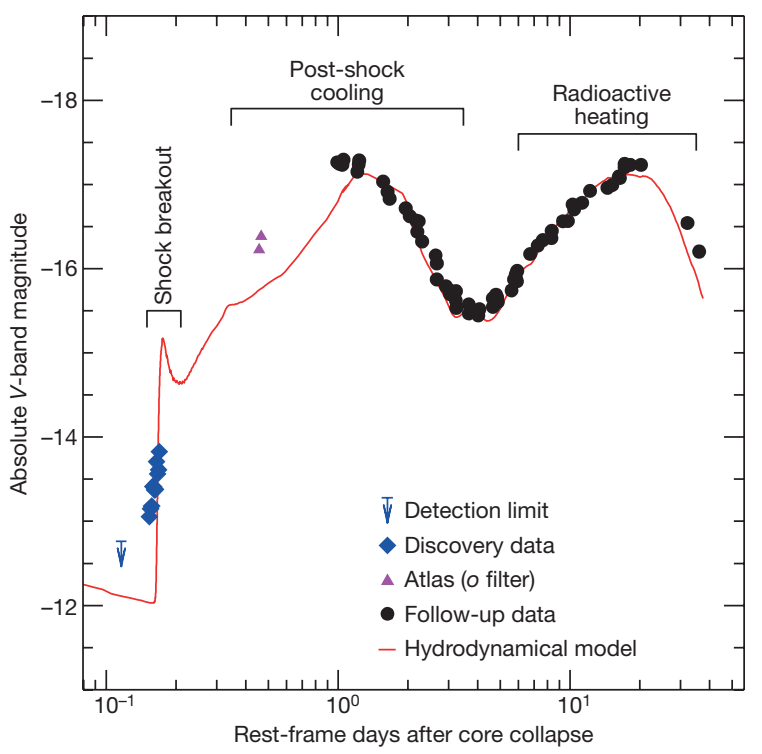

Figure 3 | Hydrodynamical model of the $V$-band light curve of SN 2016gkg. Our preferred model (red line) is able to self-consistently reproduce the observations (symbols) during three distinct phases of the supernova evolution, with different characteristic timescales and dictated by different physical properties: the shock-breakout phase, the post-shock cooling peak and the radioactivity-powered peak. Note the logarithmic scale for the time axis.

is shown in Fig. 3 (more details in Methods). The derived parameters are in close agreement with those of normal type IIb supernovae. In particular, the progenitor of SN $2016 \mathrm{gkg}$ was slightly more massive and had a more extended envelope than the well-studied type IIb supernova SN $2011 \mathrm{dh}^{23}$.

Although our model was designed to match the observed cooling and radioactive peaks, it also explains without any modification the quick rise of the discovery data as being caused by emission from the breakout of the shock. Regardless of the parameters adopted, no physical process other than the breakout of the shock can produce such a fast rise. We verified this by exploring a set of hydrostatic progenitor structures and explosion parameters and comparing the rise slopes of our light-curve models during the breakout of the shock and the postshock cooling phases (Methods). We identified the explosion energy as the dominant factor that determines the slope of the rise to the cooling peak. However, even with an energy value far beyond what is allowed by the rest of the observations, we were unable to reach a cooling peak rise rate near that of the shock-breakout phase. This result implies that different processes govern the initial rise and the cooling phase, which provides strong support for our interpretation of the early rise as the manifestation of the breakout of the shock.

A closer look at the discovery data reveals that the observed rise is more gradual than that of the model. This difference could be caused by limitations of our radiative-transfer treatment ${ }^{24,25}$, but it could also be indicative of the presence of some circumstellar material (Methods). More detailed analysis of the shock-breakout signal could potentially provide important information about the outermost progenitor structure and the physical processes that occur during the emergence of the shock. The serendipitous nature of the discovery observations and the sampling that was required highlight the difficulty of systematizing this type of finding, which has been the goal of several recent and future transient surveys, including $\mathrm{KISS}^{7}, \mathrm{HiTS}^{8}$, $\mathrm{HSC}$-SHOOT ${ }^{9}, \mathrm{KEGS}$ (http://www.mso.anu.edu.au/kegs/) and $\mathrm{ZTF}^{26}$. We note that the chance probability of this discovery is of the order of $10^{-6}$ assuming a duration of $1 \mathrm{~h}$ and one supernova per century per galaxy. If we consider other factors, such as the sky conditions of the observing site and the location of the supernova away from bright host-galaxy regions, then this probability decreases by one order of magnitude. 
Online Content Methods, along with any additional Extended Data display items and Source Data, are available in the online version of the paper; references unique to these sections appear only in the online paper.

\section{Received 18 July; accepted 7 November 2017.}

1. Langer, N. Presupernova evolution of massive single and binary stars. Annu. Rev. Astron. Astrophys. 50, 107-164 (2012).

2. Smartt, S. J. Observational constraints on the progenitors of core-collapse supernovae: the case for missing high-mass stars. Publ. Astron. Soc. Aust. 32 e016 (2015).

3. Falk, S. W. \& Arnett, W. D. Radiation dynamics, envelope ejection, and supernova light curves. Astrophys. J. Suppl. Ser. 33, 515-562 (1977)

4. Ensman, L. \& Burrows, A. Shock breakout in SN 1987A. Astrophys. J. 393 742-755 (1992).

5. Matzner, C. D. \& McKee, C. F. The expulsion of stellar envelopes in core-collapse supernovae. Astrophys. J. 510, 379-403 (1999).

6. Tominaga, N. et al. Shock breakout in type II plateau supernovae: prospects for high-redshift supernova surveys. Astrophys. J. Suppl. Ser. 193, 20 (2011).

7. Morokuma, T. et al. Kiso Supernova Survey (KISS): survey strategy. Publ. Astron. Soc. Jpn 66, 114 (2014)

8. Förster, F. et al. The High Cadence Transient Survey (HITS). I. Survey design and supernova shock breakout constraints. Astrophys. J. 832, 155 (2016).

9. Tanaka, M. et al. Rapidly rising transients from the Subaru Hyper SuprimeCam Transient Survey. Astrophys. J. 819, 5 (2016)

10. Otero, S. \& Buso, V. Discovery Certificate for Object 2016gkg. TNS Astronomical Transient Report No. 5381, https://wis-tns.weizmann.ac.il/object/2016gkg/ discovery-cert (Transient Name Server, 2016)

11. Campana, S. et al. The association of GRB 060218 with a supernova and the evolution of the shock wave. Nature 442, 1008-1010 (2006).

12. Modjaz, M. et al. From shock breakout to peak and beyond: extensive panchromatic observations of the type Ib supernova 2008D associated with Swift X-ray transient 080109. Astrophys. J. 702, 226-248 (2009).

13. Gal-Yam, A. et al. A Wolf-Rayet-like progenitor of SN $2013 \mathrm{cu}$ from spectral observations of a stellar wind. Nature 509, 471-474 (2014).

14. Garnavich, P. M. et al. Shock breakout and early light curves of type II-P supernovae observed with Kepler. Astrophys. J. 820, 23 (2016).

15. Yaron, O. et al. Confined dense circumstellar material surrounding a regular type II supernova. Nat. Phys. 13, 510-517 (2017).

16. Tartaglia, $L$ et al. The progenitor and early evolution of the type Ilb SN 2016gkg. Astrophys. J. 836, L12 (2017).

17. Arcavi, l. et al. Constraints on the progenitor of SN $2016 \mathrm{gkg}$ from its shock-cooling light curve. Astrophys. J. 837, L2 (2017)

18. Kilpatrick, C. D. et al. On the progenitor of the type Ilb supernova $2016 \mathrm{gkg}$. Mon. Not. R. Astron. Soc. 465, 4650-4657 (2017).

19. Colgate, S. A. \& McKee, C. Early supernova luminosity. Astrophys. J. 157, 623 (1969).

20. Falk, S. W. Shock steepening and prompt thermal emission in supernovae. Astrophys. J. 225, L133-L136 (1978).

21. Klein, R. I. \& Chevalier, R. A. X-ray bursts from type II supernovae. Astrophys. J. 223, L109-L112 (1978).

22. Tolstov, A. et al. Multicolor light curve simulations of population III core-collapse supernovae: from shock breakout to ${ }^{56} \mathrm{Co}$ decay. Astrophys. J. 821, 124 (2016).

23. Bersten, M. C. et al. The type Ilb supernova $2011 \mathrm{dh}$ from a supergiant progenitor. Astrophys. J. 757, 31 (2012)

24. Tolstov, A. G., Blinnikov, S. I. \& Nadyozhin, D. K. Coupling of matter and radiation at supernova shock breakout. Mon. Not. R. Astron. Soc. 429, 3181-3199 (2013)
25. Sapir, N., Katz, B. \& Waxman, E. Non-relativistic radiation mediated shock breakouts. III. Spectral properties of supernova shock breakout. Astrophys. J. 774, 79 (2013)

26. Bellm, E. The Zwicky transient facility. In The Third Hot-wiring the Transient Universe Workshop (eds Wozniak, P. R. et al.) 27-33 (2014).

27. Drout, M. R. et al. Rapidly evolving and luminous transients from PanSTARRS1. Astrophys. J. 794, 23 (2014).

28. Šimon, V., Pizzichini, G. \& Hudec, R. Evolution of the color indices in SN 2006a associated with GRB 060218. Astron. Astrophys. 523, A56 (2010).

Acknowledgements We are grateful to P. Brown for providing information about the photometry of the early Swift/UVOT data of SN 2006aj. M.C.B. acknowledges support from the Agencia Nacional de Promoción Científica y Tecnológica (ANPCyT) through grant PICT-2015-3083 'Progenitores de Supernovas de Colapso Gravitatorio' and from the Munich Institute for Astroand Particle Physics (MIAPP) of the DFG cluster of excellence 'Origin and Structure of the Universe'. M.C.B., G.F. and O.G.B. acknowledge support from grant PIP-2015-2017-11220150100746CO of CONICET 'Estrellas Binarias y Supernovas'. G.F. further acknowledges support from ANPCyT grant PICT-20152734 'Nacimiento y Muerte de Estrellas Masivas: Su relación con el Medio Interestelar'. K.M. acknowledges support from JSPS KAKENHI grant $17 \mathrm{H02864}$ Partial support for this work was provided by NASA through programmes GO14115 and AR-14295 from the Space Telescope Science Institute, which is operated by AURA, Inc., under NASA contract NAS 5-26555. M.O. acknowledges support from grant PI UNRN40B531. A.V.F. is also grateful for financial assistance from the Christopher R. Redlich Fund, the TABASGO Foundation and the Miller Institute for Basic Research in Science (University of California Berkeley). We thank the University of California Berkeley undergraduate students S. Channa, G. Halevy, A. Halle, M. de Kouchkovsky, J. Molloy, T. Ross, S. Stegman and S. Yunus for their effort in collecting Lick/Nickel data, and T.d.J. for help with some of the Keck observations. The Lick and Keck Observatory staff provided excellent assistance. A major upgrade of the Kast spectrograph on the Shane 3-m telescope at Lick Observatory was made possible through gifts from William and Marina Kast as well as the Heising-Simons Foundation. Research at Lick Observatory is partially supported by a gift from Google. KAIT and its on-going operation were made possible by donations from Sun Microsystems, Inc., the Hewlett-Packard Company, AutoScope Corporation, Lick Observatory, the NSF, the University of California, the Sylvia and Jim Katzman Foundation and the TABASGO Foundation. Some of the data presented here were obtained at the W. M. Keck Observatory, which is operated as a scientific partnership among California Institute of Technology, the University of California and NASA; the observatory was made possible by financial support from the W. M. Keck Foundation. O.G.B. is a member of the Carrera del Investigador Científico de la Comisión de Investigaciones Científicas de la Provincia de Buenos Aires (CIC), Argentina.

Author Contributions M.C.B., hydrodynamical models and interpretation. G.F., supernova and pre-supernova data analysis and interpretation. F.G., supernova data analysis and interpretation. S.V.D.D., supernova and pre-supernova data analysis and interpretation. O.G.B., binary evolution models. M.O., early data comparisons. M.T. and K.M., shock-breakout interpretation. V.B., supernova discovery. J.L.S., early supernova observations. A.V.F., Lick and Keck Observatory data and paper editing. W.Z., T.G.B., T.d.J., I.S., S.K. and N.S., observations and reductions. T.J.M., circumstellar material interpretation. K.N., pre-supernova models. S.B.C. and D.A.P., spectral reductions.

Author Information Reprints and permissions information is available at www.nature.com/reprints. The authors declare no competing financial interests. Readers are welcome to comment on the online version of the paper Publisher's note: Springer Nature remains neutral with regard to jurisdictiona claims in published maps and institutional affiliations. Correspondence and requests for materials should be addressed to M.C.B. (mbersten@fcaglp.unlp. edu.ar) and G.F. (gaston@fcaglp.unlp.edu.ar). 


\section{METHODS}

Supernova discovery data. SN 2016gkg was discovered on 2016 September $20^{10}$ by amateur astronomer V.B. using a 406-mm Skywatcher Newtonian $f=4.4$ reflector equipped with a ZWO ASI1600 MM-C camera and a clear (L) filter. Observations comprised four series of images (40,17, 20 and 21 images), each with an exposure time of $20 \mathrm{~s}$. Images were bias- and dark-subtracted, flat-fielded and aligned using the MaxIm DL software. The supernova is visible in the last three series of images at right ascension $\alpha=01 \mathrm{~h} 34 \mathrm{~min} 14.46 \mathrm{~s}$ and declination $\delta=-29^{\circ} 26^{\prime} 25.0^{\prime \prime}$ (J2000), whereas a stack of the first 40 images shows no evidence of the supernova. Clearband photometry was calibrated to standard $V$-band magnitudes on the basis of five nearby stars in the AAVSO Photometric All-Sky Survey (APASS) catalogue (https://www.aavso.org/apass). The location and catalogue magnitudes of the comparison stars are given in Extended Data Table 1 and Extended Data Fig. 1. We decided to transform magnitudes to the $V$ band because of the dense follow-up coverage in that band (including Swift/UVOT observations) compared with other bands that also lie within the range of the clear filter. In addition, as explained further below, the clear band is centred similarly to the $V$ band, which reduces the transformation error to a minimum. The results of the photometry measurements described in this section are listed in Extended Data Table 2. We note that the airmass during the complete observing time ranged between 1.00 and 1.03; thus, we expect no large systematic error in the photometry produced by differences in colour between the supernova and the comparison stars.

We estimated the detection limit on the stack of 40 images from the first series. Following ref. 29, we set the $5 \sigma$ detection limit at the magnitude level at which point sources are detected with a $50 \%$ probability. To find this magnitude, we first calibrated the $V$-band zero point of the combined image using the DAOPHOT photometry of the comparison stars. Then, we added artificial point sources in an area around the supernova location, with varying apparent magnitudes in the range of $19.0 \mathrm{mag}<V<20.0 \mathrm{mag}$. We did this in groups of 1,000 artificial stars within each 0.1 -mag bin. Finally, we used the daofind task in the DAOPHOT package of IRAF (the Image Reduction and Analysis Facility, distributed by the National Optical Astronomy Observatory, which is operated by the Association of Universities for Research in Astronomy (AURA) under cooperative agreement with the National Science Foundation (NSF); see http://iraf.noao.edu) to recover the artificial stars and thus determined the recovery fraction at each magnitude bin. We set the detection threshold $Q$ in daofind as a factor of 3-4 times the background noise level. This value was based on the image scale value of $p=0.85$ that results from the measured point-source full-width at half-maximum (FWHM) intensity of 2.1 pixels in the combined image (see figure 2 in ref. 29). By applying threshold values between 3 and 4 , we obtained consistent fractions of recovered artificial sources as a function of magnitude. The resulting detection limit was $V \approx 19.4$ mag, which is about 4.5 mag fainter than the brightness at both the cooling and nickel peaks.

To increase the signal-to-noise ratio in the last three series of images, we combined them into several groups. On the one hand, we produced a single combined image per series. On the other hand, we computed 11 averaged images from groups of 5 or 6 individual exposures (the number varied in each group owing to the different number of images in each series). Extended Data Fig. 2 shows a mosaic of the rising supernova as seen in the series of combined images. We performed point-spread function (PSF) photometry of the supernova using the DAOPHOT package. Photometric zero points were computed for each individual and combined image, using the five comparison field stars. The results are listed in Extended Data Table 2 and shown in Fig. 1. The quick initial rise is evident from both the individual-image and combined-image photometry. A linear fit to the resulting light curves yielded slopes of $37.3 \pm 5.3 \mathrm{mag} \mathrm{d}^{-1}$ using the photometry from individual exposures, $43.4 \pm 6.1 \mathrm{mag} \mathrm{d}^{-1}$ using 11 data points, and $47.2 \pm 7.8 \mathrm{mag} \mathrm{d}^{-1}$ using 3 data points. The reduced $\chi^{2}$ is about unity in all cases, which indicates that no significant departures from a linear rise in magnitudes was seen. The relatively lower slope obtained from individual measurements, although statistically compatible, can be explained by the fact that the supernova is initially very near the detection limit of individual images. This introduces a bias towards brighter measurements in DAOPHOT, which in turn causes the slope to be slightly smaller. Combined images into groups of 5 or 6 show the supernova comfortably above the detection limit. The corresponding slope is thus more reliable in these cases.

We tested our results by performing aperture photometry. We set the aperture size to approximately the value of the FWHM of the source. Although the results presented a slight systematic difference during the second series (when the supernova is the faintest), in the sense that aperture photometry yielded brighter magnitudes, the main result was confirmed: the supernova brightened by about $40 \mathrm{mag} \mathrm{d}^{-1}$. Other possible sources of error in the photometry are contamination by background host-galaxy light and unaccounted variability in the shape of the PSF across the image. The host galaxy is faint and relatively smooth at the supernova location (see Extended Data Fig. 1) and so is expected to be accurately subtracted during the measurement of the supernova count rate. Moreover, the image quality is very stable throughout the complete observations (see FWHM values in Extended Data Table 2). Therefore, any contamination would be approximately constant in time and hence would not affect the observed slope. Regarding the PSF variability, we found its shape to be consistent across the image. Also, the supernova and comparison stars are all located near the centre of the image, where the shape of the PSF is very stable.

We also tested the possible effect on the measured slope that would be caused by the supernova varying in colour during the observations. At very early times, the temperature evolution can be very fast, thus changing the supernova colour. Because we transform the measured counts in the clear band to the $V$ band, a rapid change of colour can affect the derived magnitudes. Beyond a constant error introduced by the fact that the comparison stars are expected to be redder than the supernova, the effect on the slope would arise solely from the change in supernova colour. The supernova colour during this phase is unknown. However, our models (see Methods section 'Hydrodynamical model') show that the supernova peaks at a temperature of about $2 \times 10^{5} \mathrm{~K}$ soon after explosion and cools off to about $5 \times 10^{4} \mathrm{~K}$ a few hours later. Assuming black-body spectra of varying temperature in the range $(20-200) \times 10^{3} \mathrm{~K}$, we estimated the possible variation in the calibrated $\mathrm{V}$-band supernova photometry. For this purpose we calculated an approximate clear-band transmission function, including contributions from the manufacturer's filter transmission, the quantum efficiency of the detector, two aluminium reflecting surfaces and the atmospheric transmission. This filter passband is available online (see Methods section 'Data availability'). Synthetic (clear $-V$ ) colours varied by less than $0.05 \mathrm{mag}$ in the range of temperatures considered above. Such a variation is less than $10 \%$ of the observed change in magnitude, which is below the uncertainty in the fitted slope. This means that the observed rise cannot be caused by an increasing amount of flux entering the optical range as the supernova cools down. We also estimated the size of the error introduced by the fact that the comparison stars are substantially redder than the supernova during this phase (see Extended Data Table 1). On the basis of those colours we assumed that the stars are of a spectral type between G2 and K3 and adopted ATLAS99 atmosphere models to represent their spectral energy distributions (SEDs). Synthetic colour differences between these stars and the supernova were below 0.1 mag for any assumed supernova temperature and stellar spectrum. The error is small because the clear passband, although much wider, is centred near the central wavelength of the $V$ band. The extra flux introduced by the supernova on the blue side of the filter passband is compensated by extra flux from the stars on the red side. The correction from clear to $V$ is thus accurate enough to the purposes of the current analysis.

Light curves. V.B. and J.L.S. obtained new images of SN $2016 \mathrm{gkg}$ on September 21, with clear, $B, V$ and $I$ bands. Both observers used identical telescopes and cameras. We performed aperture photometry of the supernova and comparison stars and converted the results to the standard system. The clear-band data were transformed to $V$ magnitudes. Extended Data Table 3 provides these magnitudes.

Follow-up $B, V, R$ and $I$ multiband images of SN 2016gkg were obtained with both the Katzman Automatic Imaging Telescope (KAIT) ${ }^{30}$ and the 1-m Nickel telescope at Lick Observatory. All images were reduced using a custom pipeline ${ }^{31}$ Aperture photometry was then obtained from a customized interactive data language (IDL) tool using the IDL Astronomy User's Library. Note that, owing to the small field of view for both KAIT and the Nickel telescope, we were able to use only one appropriate nearby star from the APASS catalogue as the reference star for calibration. Its magnitudes were first transformed into the Landolt system using an empirical prescription (see http://www.sdss.org/dr7/algorithms/sdssubvritransform.html\#lupton2005) and then transformed to the KAIT and Nickel natural systems. Apparent magnitudes were all measured in the KAIT4/Nickel2 natural system $^{31,32}$. The final results were transformed to the standard system using local calibrators and colour terms for KAIT4 as given in table 4 of ref. 31, and updated Nickel colour terms as given in ref. 32. Extended Data Table 3 lists the resulting standard-system magnitudes from KAIT and the Nickel telescope.

Extended Data Fig. 3 shows the resulting light curves of SN 2016gkg compared with those of the well-observed type IIb supernovae SN 1993J and SN 2011dh. In this figure we also added the early-time $V$-band (or transformed) photometry from Atlas, ASAS, LCOGT and Swift.

Spectra. Spectroscopic observations of SN 2016gkg were performed using the Kast spectrograph on the Lick Observatory 3-m Shane telescope on 2016 September 24, November 3, December 4 and December 23, the Low Resolution Imaging Spectrometer (LRIS) ${ }^{33}$ on the 10-m Keck-I telescope on 2016 September 28 and 2017 January 2, and the Deep Imaging Multi-Object Spectrograph (DEIMOS) ${ }^{34}$ on the 10 -m Keck-II telescope on 2016 October 25 with both the 600 and 1,200 lines $\mathrm{mm}^{-1}$ gratings. Data were obtained at the parallactic angle ${ }^{35}$ to ensure accurate relative spectrophotometry; moreover, LRIS is equipped with an atmospheric dispersion corrector. Standard data reduction (including bias subtraction, flat-fielding and spectral extraction) was performed within IRAF. The spectra were flux calibrated via observations of spectrophotometric standard stars at similar airmasses to those 
for the supernova observations. The spectra are shown in Extended Data Fig. 3 Also displayed for comparison are spectra of the type IIb supernovae SN 1993 ${ }^{36,37}$ and SN $2011 \mathrm{dh}^{38}$; these spectra were all obtained from WISeREP ${ }^{39}$. We find, generally, that the SN $2016 \mathrm{gkg}$ spectra bear a stronger resemblance to those of SN 2011dh than to those of SN 1993J.

We produced synthetic spectra using the SYNOW code (see ref. 40 and references therein) with the aim of estimating expansion velocities as a function of phase. SYNOW provides approximate continuum and line-strength levels, using simple assumptions. It can, however, provide a robust estimate of the velocity at the photosphere from the overall fit to the observed spectrum including lines of several ionic species. Each SYNOW spectrum was computed consistently with the rest of the epochs, by keeping a smooth variation of the photospheric temperature and velocity with epoch. The derived photospheric velocities, shown in Extended Data Fig. 4b, were used to compare with the hydrodynamical models

Hydrodynamical model. To analyse the supernova photometry and photospheric velocity evolution, we used a one-dimensional Lagrangian hydrodynamics code that assumes flux-limited radiation diffusion for optical photons and a one-group approximation for the non-local deposition of rays produced by radioactive decay $^{41}$. The code simulates the supernova explosion by injecting energy in a hydrostatic structure and self-consistently following the shock-wave propagation inside the star, the shock breakout and the subsequent expansion of the supernova ejecta during the photospheric phase. For this work the code was adapted to include light-travel time effects and limb-darkening corrections, following the prescription of ref. 4 , which are relevant only at the earliest epochs (the first $2 \mathrm{~h}$ ) of the supernova evolution.

As initial configurations (pre-supernova models), we used hydrostatic structures from single stellar-evolution calculations. Specifically, models with pre-supernova masses of $3.3 M_{\odot}(\mathrm{HE} 3.3), 4 M_{\odot}(\mathrm{HE} 4), 5 M_{\odot}$ (HE5) and $6 M_{\odot}$ (HE6), which correspond to initial main-sequence masses of $13 M_{\odot}, 15 M_{\odot}, 18 M_{\odot}$ and $20 M_{\odot}$ respectively, were tested ${ }^{42}$. All of these configurations are compact hydrogen-free structures with radii of $R<3 R_{\odot}$, and were evolved from He burning until core collapse, assuming solar initial abundances ${ }^{42}$. However, these pre-supernova models were later modified to take into account the presence of a tenuous hydrogen envelope (of at most $1 M_{\odot}$ ). This model construction is necessary to reproduce successfully the two-peak morphology of the light curves and the spectra of type IIb supernovae (see more details in ref. 23).

Our first step in the modelling was to find a set of parameters, such as explosion energy $(E)$, ejected mass $\left(M_{\mathrm{ej}}\right)$, and the mass of synthesized ${ }^{56} \mathrm{Ni}\left(M_{\mathrm{Ni}}\right)$ and its distribution, that provided a good representation of the main bolometric light-curve peak and the photospheric velocity $\left(v_{\mathrm{ph}}\right)$ evolution. Owing to large uncertainties in distance, reddening, bolometric corrections, photospheric velocities, and so on, we do not attempt a statistical fit to the observations. However, our conclusions are not affected by this. The model that provides the best overall agreement with the bolometric light curve and expansion velocities, shown with a solid line in Extended Data Fig. 4a, b, corresponds to the HE5 model for an explosion energy of $E=1.2 \times 10^{51} \mathrm{erg}$, a ${ }^{56} \mathrm{Ni}$ mass of $M_{\mathrm{Ni}}=0.085 M_{\odot}$ and an ejecta mass of $M_{\mathrm{ej}}=3.4 M_{\odot}$, assuming the formation of a compact remnant $\left(M_{\text {cut }}\right)$ of $1.6 M_{\odot}$. Extended Data Fig. 4 also shows models with lower and higher mass that resulted in worse fits to the data. Note that the HE4 model, which corresponds to $M_{\mathrm{ej}}=2.5 M_{\odot}, E=1 \times 10^{51} \mathrm{erg}, M_{\mathrm{Ni}}=0.087 M_{\odot}$ and $M_{\mathrm{cut}}=1.5 M_{\odot}$, provides a possible solution, although slightly worse than that of HE5. Indeed, models with intermediate parameters-between those of HE4 and HE5, with $M_{\mathrm{ej}}=(2.5-3.4) M_{\odot}, E=(1-1.2) \times 10^{51}$ erg and $M_{\mathrm{Ni}}=(0.085-0.087) M_{\odot}-$ are also valid. Therefore, these values can be considered as the ranges of validity for the physical parameters. An important conclusion of this analysis is that the progenitor of SN $2016 \mathrm{gkg}$ needs to be a relatively low-mass He star, as is commonly the case for stripped-envelope supernovae ${ }^{43-45}$.

Once the global parameters were set, we focused on the modelling at epochs before the onset of radioactive-decay domination. At such times, the light curve is extremely sensitive to the extent (radius) and mass of the H-rich envelope. Therefore, we modified the initial structure in the HE5 model by smoothly attaching a low-mass $\mathrm{H}$-rich envelope in hydrostatic and thermal equilibrium. Note that the HE4 model could also have been used in this analysis without changing the conclusions. Different configurations were tested with various progenitor radii and envelope masses. By comparing with the observations, we were able to find our preferred configuration, denoted as the preferred model and shown in Fig. 3, corresponding to an object with an $\mathrm{H}$-rich envelope of radius $R=320 R_{\odot}$ and a mass of $M_{\mathrm{env}}=0.01 M_{\odot}$. The preferred model provides a satisfactory match to the observations. Although the agreement during the post-shock cooling peak is not perfect, the model consistently reproduces the complete supernova evolution. Extended Data Fig. 4c shows this model with a solid line, compared with models of larger (smaller) progenitor radii, which overestimate (underestimate) the luminosity during the post-shock cooling phase. From these comparisons we conclude that reasonable ranges of validity for the radius and mass of the envelope are $R=(300-340) R_{\odot}$ and $M_{\mathrm{env}}=(0.01-0.09) M_{\odot}$. Note that this analysis was based on the $V$-band light curve instead of the bolometric luminosity. The reason for using the $V$-band data was that the earliest observations were done with that band or with a clear filter that was transformed to the $V$ band. In addition, a bolometric correction at such early epochs is highly uncertain. Therefore, we computed theoretical $V$-band photometry assuming a black-body SED at the thermalization temperature ${ }^{23}$.

Previous analyses of the post-shock cooling emission of SN 2016gkg arrived at different values of $R$ and $M_{\text {env }}$, for example, $R \approx 257 R_{\odot}$ (ref. 16), $R=(50-125) R_{\odot}$ (ref. 17) and $R=(44-131) R_{\odot}$ (ref. 18). In these analyses an analytical approach was used, which is simpler than the hydrodynamical modelling presented here. Moreover, such analytic approaches have been proven to be wrong in the interpretation of the progenitor radius for the similar type IIb supernova SN $2011 \mathrm{dh}^{23,46}$. By contrast, a similar hydrodynamical model for the post-shock cooling emission ${ }^{47}$ of SN 2016gkg yielded a radius of $R \approx 200 R_{\odot}$ and an envelope mass of $M_{\mathrm{env}} \approx 0.02 M_{\odot}$, which are smaller than our values. Although the hydrodynamical code used ${ }^{47}$ is similar to ours, the initial structures are not: instead, parametric models were used that allow the modification of the initial density profiles to take into account the existence of a tenuous extended envelope. This method, contrary to ours, does not ensure hydrostatic and thermal equilibrium of the external envelope. This could be a cause of the differences in the derived parameters. Several interesting conclusions can be derived from Extended Data Fig. 4, as follows.

(1) Our model indicates that the optical light curves of type IIb supernova have three peaks, rather than the double peaks usually referred to in the literature. These are the shock-breakout (SBO) peak, the post-shock cooling peak and the nickel-powered peak (see also Fig. 3).

(2) All models indicate that the discovery data can be interpreted as the SBO, regardless of the progenitor radius adopted. This is still valid for the values proposed previously ${ }^{18,47}$.

(3) The progenitor radius has a much more noticeable effect on the decline rate than on the rise rate during the cooling peak.

(4) The larger the progenitor radius, the more luminous the cooling peak becomes and the later it appears.

To further test our SBO interpretation of the early-time data, we extensively explored parameters other than the radius that could increase the slope of the cooling peak to a similar rate to that of the SBO peak (that is, around $40 \mathrm{mag} \mathrm{d}^{-1}$ ). We found that the explosion energy has the strongest effect on increasing this slope (Extended Data Fig. 4d). However, within the range of $E$ values allowed by the modelling of the complete supernova evolution, the cooling-peak rise is always appreciably slower than the SBO rise. This is true even in an extreme case, with an explosion energy of $5 \times 10^{51} \mathrm{erg}$ (about four times larger than our preferred model), as shown in Extended Data Fig. 5. Our analysis demonstrates that the initial rise is always steeper than the rise to the post-shock cooling peak, and that there is always a local maximum in the light curve between both phases, provided that realistic pre-supernova structures are assumed. This result gives support to our SBO interpretation.

Remarkably, we have demonstrated a model that is able to reproduce three distinct light-curve phases with very dissimilar timescales (note the logarithmic timescale in Fig. 3), assuming a standard set of parameters that fit normal type IIb supernovae. Nevertheless, a close look at the earliest phases shows that the observed rise is slower than that of the models during the SBO phase (see Extended Data Fig. 5). We thus tested if this situation could be ameliorated by adding some extra surrounding material to the initial density structure. We did not assume this material to be in hydrostatic equilibrium. It could be material ejected by the progenitor before the supernova explosion, probably in the form of a dense wind. Extended Data Fig. 5 shows a model with such circumstellar material (CSM; dashed line). Clearly, the presence of this material slows the rise during the SBO, in better concordance with the observations, without affecting the light curve at later epochs (at times of 1 day or more), owing to the small amount of matter involved. Specifically, the model presented here corresponds to a mass of $0.002 M_{\odot}$ distributed out to $3 \times 10^{13} \mathrm{~cm}$, assuming a steep power-law density profile with an index of 10 . This corresponds to an average mass-loss rate of $6 \times 10^{-4} M_{\odot} \mathrm{yr}^{-1}$, for a wind velocity of $v_{\text {wind }}=100 \mathrm{~km} \mathrm{~s}^{-1}$. However, we found that the exact properties of this material are not very relevant. In fact, assuming a range of other density structures with different slopes and extensions produces similar results. In particular, for a constant wind profile (with an index of $r^{-2}$ ), we found almost the same results, but in this case the mass-loss rate increased by almost two orders of magnitude. On the basis of our tests, we can say that the mere presence of this material is enough to slow down the SBO rise, with very little dependence on its exact nature. In this context, it is noteworthy that recently there has been increasing evidence from early-time observations of normal supernovae (photometry and spectroscopy) of the existence of surrounding material in the vicinity of the progenitor, 
possibly produced by a dense wind or an eruption that occurred shortly before the explosion ${ }^{13,15,48,49}$.

It has been noted that non-thermal processes could have a role in shaping the light curve during the SBO phase ${ }^{25}$. This second-order effect could smooth the SBO peak. However, according to those calculations, the initial rise rate remains basically unchanged. This suggests that, even if deriving detailed CSM properties from our models would be premature, the conclusion of the SBO signal detection would remain well founded. A deeper analysis of these effects is thus left for future study.

Previous SBO claims. Some supernovae have been associated in the past with possible SBO emission. The outstanding cases of SN 2006aj and SN 2008D ${ }^{11,12,50,51}$ are worth noting. SN 2006aj was connected with a $\gamma$-ray burst (GRB) and SN 2008D was preceded by an X-ray flash. The X-ray transient in both cases was interpreted by some as being produced by the SBO. However, the peculiar characteristics of both events and the lack of a model that fully describes the hard and soft emission cast some doubt on this interpretation ${ }^{51-53}$. The early-time optical data for these supernovae are shown in Fig. 2. The initial optical rise of SN 2008D has a slope and luminosity similar to those of SN 2016gkg during the cooling peak. Interestingly, Swift/UVOT $V$-band data for SN 2006aj ${ }^{11}$ obtained within $1 \mathrm{~h}$ after the associated GRB show a similarly steep rise of $49 \pm 16 \mathrm{mag} \mathrm{d}^{-1}$ as that of SN $2016 \mathrm{gkg}$ at discovery, although with a much larger luminosity. Re-analysis of the early-time data based on the 2015 measurements available in the Swift Optical/Ultraviolet Supernova Archive (SOUSA; ref. 54 and P. Brown, private communication) provides a smaller slope of $21 \pm 12 \mathrm{mag} \mathrm{d}^{-1}$. Nevertheless, the behaviour of this emission may still be interpreted as an SBO, although emerging from some CSM instead of the stellar surface. It should also be noted that the rise slope of the SBO in this case may be reduced by a declining contribution from the GRB afterglow.

More recently, the case of KSN 2011d, discovered by the Kepler mission, was considered an SBO detection on the basis of an excess in the early-time optical light curve relative to a simple analytic model ${ }^{14}$. However, modifying the data binning and comparison function shows ${ }^{55}$ that there is no statistical significance for an SBO in KSN 2011d.

Progenitor candidate. The SN $2016 \mathrm{gkg}$ site is contained in publicly available archival HST images, obtained with the Wide-Field Planetary Camera 2 (WFPC2) in bands F450W, F606W and F814W on 2001 August 21, as part of programme GO-9042. We also obtained images of the supernova itself on 2016 October 10 with HST, with the Wide-Field Camera 3 (WFC3) UVIS channel in band F555W, as part of the Target of Opportunity (ToO) programme GO-14115. The observations consisted of 24 dithered frames, each of 10-s duration; the short exposure time mitigated against possible saturation by a potentially bright supernova (we knew that the supernova brightness was increasing at the time, but not to what level). The frames were combined into a final mosaic of 240-s total exposure using AstroDrizzle ${ }^{56}$ in DrizzlePac ${ }^{57}$ within PyRAF. Adopting 13 stars in common between the WFPC2 image mosaic at F606W and the WFC3/UVIS mosaic at F555W, we astrometrically registered the two datasets with a root-mean-square (r.m.s.) uncertainty of 0.42 WFPC2/WF pixels $\left(0.042^{\prime \prime}\right.$; see the registered images in Extended Data Fig. 6). After measuring the centroid of the supernova in the WFC3 mosaic, we found that the supernova position on the WFPC2 mosaic is $(1552.47,196.39)$. On the WFPC2 mosaic we measured a centroid for the progenitor candidate of $(1552.63,196.06)$. This is a difference of 0.37 pixels, which is within the astrometric uncertainty. We therefore consider this progenitor candidate to be solidly identified; it will not be until the candidate has vanished wel after the supernova explosion that its identity will be confirmed with little doubt. We note that there is more ambiguity in the identifications of the candidates in refs 16 and 18 than in ours.

We measured photometry for the progenitor candidate running Dolphot ${ }^{58}$ on the individual ' $c 0 \mathrm{~m}$ ' WFPC2 frames after masking cosmic rays with AstroDrizzle. Although the star is relatively isolated, with little apparent background, we set FitSky $=3$ (rather than FitSky=1), because the star is only 10 pixels from the edge of the WF4 chip and much of the sky annulus would sit off the edge. We therefore also set RAper $=8$, as well as InterpPSFlib $=1$ using the TinyTim PSF library. We enabled charge transfer efficiency corrections by setting WFPC2UseCTE $=1$ in Dolphot. This resulted in brightnesses on the Vega system of $m_{\mathrm{F} 450 \mathrm{~W}}=24.07 \pm 0.16 \mathrm{mag}, m_{\mathrm{F} 606 \mathrm{~W}}=24.04 \pm 0.07 \mathrm{mag}$ and $m_{\mathrm{F} 814 \mathrm{~W}}=23.58 \pm 0.14 \mathrm{mag}$. The authors of ref. 16 found $23.60 \pm 0.14 \mathrm{mag}$, $23.72 \pm 0.08 \mathrm{mag}$ and $23.25 \pm 0.14 \mathrm{mag}$, respectively, for their 'object $\mathrm{A}^{\prime}$ (which we have shown is the progenitor candidate). In addition, in ref. 16, results from ref. 18 were converted from STMAG to VEGAMAG, yielding $23.42 \mathrm{mag}$, $23.10 \mathrm{mag}$ and $23.32 \mathrm{mag}$, respectively, and it is noted that this object in the Hubble Source Catalogue has $23.85 \pm 0.08 \mathrm{mag}$ at F450W and $23.34 \pm 0.05 \mathrm{mag}$ at F606W, all VEGAMAG. Our measurements are brighter by $0.3-0.4$ mag compared to those of ref. 16. After contacting the authors of ref. 16, we now recognize that the source of the differences is the cosmic-ray masking procedure (they used
LACosmic whereas we used AstroDrizzle) and the combination of the FitSky/ RAper parameters in Dolphot (we used 3/8 whereas they used 1/4).

From our photometry of the progenitor candidate, and assuming a distance modulus of $32.11 \pm 0.38 \mathrm{mag}$ and an extinction (Milky Way only) of $A_{V}=0.053 \mathrm{mag}$ (see Methods section 'Supernova site extinction and metallicity'), we obtained absolute magnitudes of $M_{\mathrm{F} 450 \mathrm{~W}}=-8.1 \pm 0.4 \mathrm{mag}, M_{\mathrm{F} 606 \mathrm{~W}}=-8.1 \pm 0.4 \mathrm{mag}$ and $M_{\mathrm{F} 814 \mathrm{~W}}=-8.6 \pm 0.4 \mathrm{mag}$. We performed $\chi^{2}$ fits of the resulting SED to stellar atmosphere models from ATLAS9 $9^{59}$ and found best-fit values of $T_{\text {eff }}=7,250_{-850}^{+900} \mathrm{~K}$ and luminosity $\log \left(L / L_{\odot}\right)=5.10_{-0.19}^{+0.17}$. The fitted SED is shown in Extended Data Fig. 6. Assuming a black body, this corresponds to a progenitor radius of $226_{-73}^{+98} R_{\odot}$. Such a radius is slightly smaller than (but still compatible with) what we estimated from the hydrodynamical modelling (see refs 16,18 and 47 for previous estimates). Progenitor model. We attempted to find a consistent progenitor picture based on the information from the light-curve modelling and the pre-explosion photometry. The location of the pre-explosion object in the Hertzsprung-Russell diagram (HRD) is not compatible with the endpoints of single stellar evolutionary tracks ${ }^{16}$, unless some non-standard enhanced mass loss is assumed. The relatively low progenitor mass derived from the light-curve modelling, however, goes against the possibility of large mass loss produced by an isolated star wind. A more natural scenario is that of a close binary system in which the primary star explodes as a supernova after transferring mass to its companion. This type of system allows for strong mass loss even in the case of relatively low-mass progenitors. Here we present a possible such scenario for SN 2016gkg. Our proposed model is not supposed to be a unique solution.

We used a code described ${ }^{60}$ and applied ${ }^{61}$ previously to SN 2011dh. This code has detailed and updated physical ingredients (see ref. 61 and references therein). When stars are detached, it works as a standard Henyey code. When the donor star undergoes Roche-lobe overflow ${ }^{62}$, the code computes the mass-transfer rate simultaneously with the structure of the donor star in an implicit Henyeylike, numerically stable algorithm. We neglected rotation of the components and assumed that the orbit is circularized and synchronized. We assumed that the accreting star retains a fraction $\beta$ of the material transferred by the donor component $-a$ free parameter the value of which is kept constant throughout the entire evolution. Here we considered values of $\beta=0.0,0.25$ and 0.50 (that is, nonconservative evolution). The material lost from the system is assumed to have the specific angular momentum of the companion star.

We found good agreement with the observations by assuming a binary progenitor with solar abundance, initial masses of $19.5 M_{\odot}$ and $13.5 M_{\odot}$ and an orbital period of 70 days. The primary star explodes as an supernova, with a final mass and radius of $M=4.61 M_{\odot}$ and $R=183 R_{\odot}$ and a final orbital period of 631 days. The total amount of hydrogen that remains in the primary is $6 \times 10^{-3} M_{\odot}$, contained in the outer approximately $0.06 M_{\odot}$ of the star. The surface abundance is $X_{\text {surf }}=0.21$. The model stays inside the error box of Extended Data Fig. $6 \mathrm{~d}$ for the final $14,000 \mathrm{yr}$ of evolution. All of these values are almost independent of the uncertain value of $\beta$.

Qualitatively, the evolution of the progenitor of SN 2016gkg is very similar to that of SN 2011dh. For both objects, binary models are more plausible candidates than single stars, because isolated objects need very specific mass-loss rates to account for the final luminosity and effective temperature ( $L$ and $\left.T_{\text {eff }}\right)$ indicated by the pre-supernova observations. In binary systems, the donor (progenitor) star spends almost all of its final nuclear burning stages (carbon, neon, oxygen and silicon), which last for several thousand years, under Roche-lobe-overflow conditions. This places the progenitor in a well-defined region of the HRD (Extended Data Fig. 6), inside the error box in $\left(L, T_{\text {eff }}\right)$ for SN 2016gkg. Thus, binary systems provide a natural reference frame for interpreting the evolution of the progenitor of SN $2016 \mathrm{gkg}$.

We note that in ref. 18 a very different progenitor was proposed, with $15 M_{\odot}+1.5 M_{\odot}$ and $P_{\text {ini }}=1,000$ days, which yielded a pre-supernova mass of $5.2 M_{\odot}$. The evolutionary track in ref. 18 is completely different from ours, because it undergoes core He burning as a red supergiant, and after He exhaustion executes very large loops. This scenario requires some fine tuning of the initial conditions for the pre-supernova model to be at the observed location in the HRD; as discussed above, ours does not.

Remarkably, for the computed systems, most of the mass accreted by the companion star is gained before core He burning. Thus, there is plenty of time for the accreted mass to accommodate to the stellar structure. This implies that the companion star remains close to the zero-age main sequence in the HRD, while being over-luminous for its mass. Similarly to those for SN 2011dh, our calculations predict the existence of a hot companion to the progenitor that should remain after the explosion. The position of the companion star in the HRD depends on its final mass and therefore on $\beta$. At the moment of the explosion, the companion star is still undergoing core hydrogen burning. The presence of this object may be tested with future observations, once the supernova fades sufficiently. 
Supernova site extinction and metallicity. To estimate a colour excess for SN 2016gkg, we compared its $(B-V)$ colours with those of SN 2011 dh, which shows very similar spectral evolution (see Extended Data Fig. 3). Adopting only the Galactic reddening of $E(B-V)=0.017 \mathrm{mag}$ for SN 2016gkg, and a total reddening of $E(B-V)=0.074 \mathrm{mag}$ for SN $2011 \mathrm{dh}^{38}$, the colour curves of both supernovae match very well. This indicates that the host-galaxy reddening for the former supernova is negligible.

To test this, we inspected the optical spectra for signatures of dust extinction by looking for interstellar absorption lines. In our highest-resolution spectrum, obtained with DEIMOS on 2016 October 25, we detected the Na I D doublet both from the Milky Way and at the redshift of the host galaxy. The equivalent width of the $\mathrm{D} 1+\mathrm{D} 2$ lines was $0.16+0.13 \AA$ for the Galactic component and $0.43+0.26 \AA$ for the host-galaxy component. This may indicate a larger host extinction than that from the Milky Way (as assumed in ref. 17). However, the Na I D equivalent width has been shown to be a poor indicator of dust extinction ${ }^{63}$. Following ref. 63, we instead studied the diffuse interstellar band at $5,780 \AA$. Such a feature is not detected in our DEIMOS spectrum, with a limiting equivalent width of around $0.01 \AA$. This is indicative of a low host-galaxy extinction, $A_{V}<0.05 \mathrm{mag}$.

On the basis of the colour comparison with SN $2011 \mathrm{dh}$ and the study of spectral lines, we decided to neglect host-galaxy extinction; our results are not affected by this assumption.

We can also estimate the metallicity more directly from the spectrum of an $\mathrm{H}$ II region, at $\alpha=01 \mathrm{~h} 34 \mathrm{~min} 14.53 \mathrm{~s}, \delta=-29^{\circ} 26^{\prime} 16.4^{\prime \prime}$ (J2000), which is about $8.6^{\prime \prime}$ nearly due north of the supernova site ${ }^{64}$. We had included the $\mathrm{H}$ II region in the slit, while observing the supernova with Keck/DEIMOS on 2016 October 25 with the lower-resolution grating. Owing to the short DEIMOS slit length, for strong emission lines (in this case, $\mathrm{H}$ ) not much spatial area exists on the spectral image for accurately estimating the overall night-sky value, which probably introduces systematic uncertainty in the strong-line flux. Nonetheless, we measured the Balmer decrement from the observed spectrum and estimate $A_{V}=3.5 \mathrm{mag}$ for the nebula. The line-of-sight Galactic foreground contribution to the extinction is comparatively low, $A_{V}=0.053 \mathrm{mag}$ (ref. 65). A high extinction is plausible, given the conspicuous presence of a counterpart of the $\mathrm{H}$ in region in archival Spitzer Space Telescope data. The nebula corresponds to a luminous source at both $3.6 \mu \mathrm{m}$ and $4.5 \mu \mathrm{m}$, and one of the brightest sources at $24 \mu \mathrm{m}$, in the outer disk of the galaxy. We corrected the spectrum for this extinction, and for an assumed recession velocity of $1,481 \mathrm{~km} \mathrm{~s}^{-1}$ (from the NASA/IPAC Extragalactic Database, NED), and show the corrected spectrum in Extended Data Fig. 6.

We enlisted the various strong-line indicators used to estimate the metallicity of extragalactic H II regions; these lines are labelled in Extended Data Fig. 6e. We measured their fluxes from the corrected spectrum and list them in Extended Data Table 4. Unfortunately, the spectrum did not go blueward enough that we could use the well-calibrated $\mathrm{R}_{23}$ indicator or [ $\mathrm{N}$ II]/[O II], which depends on the intensity of the $\left[\mathrm{O}_{\mathrm{II}}\right] \lambda 3,727$ line $^{66}$. Instead, we had to rely on other indicators. From the indicators $\mathrm{R}_{3}, \mathrm{~N}_{2}$ and $\mathrm{S}_{2}$, as defined in ref. 67, and using the 'S calibration' (equation (6) in ref. 67), we find $12+\log (\mathrm{O} / \mathrm{H})=8.65$. Considering the indicators $\mathrm{R}_{3}, \mathrm{~N}_{2}$ and $\mathrm{O}_{3} \mathrm{~N}_{2}$ from ref. 68, derived with the online tool at http://www.arcetri.astro.it/ metallicity/, we arrive at $12+\log (\mathrm{O} / \mathrm{H})=8.7$.

Culling all of these estimates and assuming a solar abundance, $12+\log (\mathrm{O} / \mathrm{H})=8.69 \pm 0.05$ (ref. 69), the metallicity at the SN 2016gkg site appears to be consistent with solar, and we have adopted this throughout.

Data availability. The datasets analysed during this study are available from http:// fcaglp.unlp.edu.ar/ gaston/data/sn2016gkg/.

Code availability. We have opted not to make the supernova light-curve modelling code or the binary evolution code available because they have not been prepared for portability and lack the necessary documentation for general use. However, al optical spectra will be made available at WISeREP ${ }^{39}$.

29. Harris, W. E. A comment on image detection and the definition of limiting magnitude. Publ. Astron. Soc. Pacif. 102, 949-953 (1990)

30. Filippenko, A. V., Li, W. D., Treffers, R. R. \& Modjaz, M. The Lick Observatory supernova search with the Katzman Automatic Imaging Telescope. ASP Conf. Ser. 246, 121-130 (2001).

31. Ganeshalingam, M. et al. Results of the Lick Observatory supernova search follow-up photometry program: BVRI light curves of 165 type la supernovae. Astrophys. J. Suppl. Ser. 190, 418-448 (2010).

32. Shivvers, I. et al. The nearby type Ibn supernova 2015G: signatures of asymmetry and progenitor constraints. Mon. Not. R. Astron. Soc. 471, 4381-4397 (2017)

33. Oke, J. B. et al. The Keck Low-Resolution Imaging Spectrometer. Publ. Astron. Soc. Pacif. 107, 375-385 (1995).

34. Faber, S. M. et al. The DEIMOS spectrograph for the Keck II telescope: integration and testing. Proc. SPIE 4841, 1657-1669 (2003)

35. Filippenko, A. V. The importance of atmospheric differential refraction in spectrophotometry. Publ. Astron. Soc. Pacif. 94, 715-721 (1982).
36. Barbon, R. et al. SN 1993J in M 81: one year of observations at Asiago. Astron. Astrophys. Suppl. Ser. 110, 513-519 (1995).

37. Matheson, T. et al. Optical spectroscopy of supernova 1993J during its first 2500 days. Astron. J. 120, 1487-1498 (2000).

38. Ergon, M. et al. Optical and near-infrared observations of SN $2011 \mathrm{dh}$ - the first 100 days. Astron. Astrophys. 562, A17 (2014)

39. Yaron, O. \& Gal-Yam, A. WISeREP-an interactive supernova data repository. Publ. Astron. Soc. Pacif. 124, 668-681 (2012).

40. Branch, D. et al. Direct analysis of spectra of type lb supernovae. Astrophys. J. 566, 1005-1017 (2002)

41. Bersten, M. C., Benvenuto, O.\& Hamuy, M. Hydrodynamical models of type II plateau supernovae. Astrophys. J. 729, 61 (2011).

42. Nomoto, K. \& Hashimoto, M. Presupernova evolution of massive stars. Phys. Rep. 163, 13-36 (1988).

43. Drout, M. R. et al. The first systematic study of type lbc Supernova multi-band light curves. Astrophys. J. 741, 97 (2011).

44. Lyman, J. D. et al. Bolometric light curves and explosion parameters of 38 stripped-envelope core-collapse supernovae. Mon. Not. R. Astron. Soc. 457, 328-350 (2016)

45. Taddia, F. et al. The Carnegie Supernova Project I: analysis of stripped-envelope supernova light curves. Astron. Astrophys. https://doi.org/10.1051/00046361/201730844 (2017).

46. Van Dyk, S. D. et al. The progenitor of supernova 2011 dh has vanished. Astrophys. J. 772, L32 (2013).

47. Piro, A. L. et al. Numerically modeling the first peak of the type Ilb SN 2016gkg. Astrophys. J. 846, 94 (2017)

48. Khazov, D. et al. Flash spectroscopy: emission lines from the ionized circumstellar material around $<10$-day-old type II supernovae. Astrophys. J. 818, 3 (2016).

49. Moriya, T. J., Yoon, S.-C., Gräfener, G. \& Blinnikov, S. I. Immediate dense circumstellar environment of supernova progenitors caused by wind acceleration: its effect on supernova light curves. Mon. Not. R. Astron. Soc. 469, L108-L112 (2017)

50. Soderberg, A. M. et al. An extremely luminous X-ray outburst at the birth of a supernova. Nature 453, 469-474 (2008).

51. Mazzali, P. A. et al. The metamorphosis of supernova SN 2008D/XRF 080109: a link between supernovae and GRBs/hypernovae. Science 321, 1185-1188 (2008).

52. Ghisellini, G., Ghirlanda, G. \& Tavecchio, F. Did we observe the supernova shock breakout in GRB 060218? Mon. Not. R. Astron. Soc. 382, L77-L81 (2007)

53. Li, L.-X. The X-ray transient 080109 in NGC 2770: an X-ray flash associated with a normal core-collapse supernova. Mon. Not. R. Astron. Soc. 388, 603-610 (2008).

54. Brown, P. J., Breeveld, A. A., Holland, S., Kuin, P. \& Pritchard, T. SOUSA: the Swift Optical/Ultraviolet Supernova Archive. Astrophys. Space Sci. 354, 89-96 (2014).

55. Rubin, A. \& Gal-Yam, A. Exploring the efficacy and limitations of shock-cooling models: new results for type II supernovae observed by the Kepler mission. Astrophys. J. 848, 8 (2017).

56. Fruchter, A. S., Hack, W., Dencheva, N., Droettboom, M. \& Greenfield, P. BetaDrizzle: a redesign of the MultiDrizzle package. In 2010 Space Telescope Science Institute Calibration Workshop 382-387 (2010).

57. Gonzaga, S., Hack, W., Fruchter, A. \& Mack, J. (eds) The DrizzlePac Handbook (STScl, 2012)

58. Dolphin, A. E. WFPC2 stellar photometry with HSTPHOT. Publ. Astron. Soc. Pacif 112, 1383-1396 (2000)

59. Kurucz, R. ATLAS9 Stellar Atmosphere Programs and $2 \mathrm{~km} / \mathrm{s}$ Grid. Kurucz CD-ROM No. 13 (Smithsonian Astrophysical Observatory, 1993).

60. Benvenuto, O. G. \& De Vito, M. A. A code for stellar binary evolution and its application to the formation of helium white dwarfs. Mon. Not. R. Astron. Soc 342, 50-60 (2003)

61. Benvenuto, O. G., Bersten, M. C. \& Nomoto, K. A binary progenitor for the type Ilb supernova 2011dh in M51. Astrophys. J. 762, 74 (2013).

62. Paczýnski, B. Evolutionary processes in close binary systems. Annu. Rev. Astron Astrophys. 9, 183 (1971).

63. Phillips, M. M. et al. On the source of the dust extinction in type la supernovae and the discovery of anomalously strong Na I absorption. Astrophys. J. 779, 38 (2013).

64. Koopmann, R. A. \& Kenney, J. D. P. An atlas of $H$ and R images and radial profiles of bright isolated spiral galaxies. Astrophys. J. Suppl. Ser. 162, 97-112 (2006).

65. Schlafly, E. F. \& Finkbeiner, D. P. Measuring reddening with Sloan Digital Sky Survey stellar spectra and recalibrating SFD. Astrophys. J. 737, 103 (2011).

66. Kewley, L. J. \& Dopita, M. A. Using strong lines to estimate abundances in extragalactic H II regions and starburst galaxies. Astrophys. J. Suppl. Ser. 142, 35-52 (2002).

67. Pilyugin, L. S. \& Grebel, E. K. New calibrations for abundance determinations in H II regions. Mon. Not. R. Astron. Soc. 457, 3678-3692 (2016).

68. Curti, M. et al. New fully empirical calibrations of strong-line metallicity indicators in starforming galaxies. Mon. Not. R. Astron. Soc. 465, 1384-1400 (2017).

69. Asplund, M., Grevesse, N., Sauval, A. J. \& Scott, P. The chemical composition of the Sun. Annu. Rev. Astron. Astrophys. 47, 481-522 (2009).

70. Richmond, M. W. et al. UBVRI photometry of SN 1993J in M81: the first 120 days. Astron. J. 107, 1022-1040 (1994).

71. Arcavi, I. et al. SN $2011 \mathrm{dh}$ : discovery of a type llb supernova from a compact progenitor in the nearby galaxy M51. Astrophys. J. 742, L18 (2011). 


\section{RESEARCH LETTER}

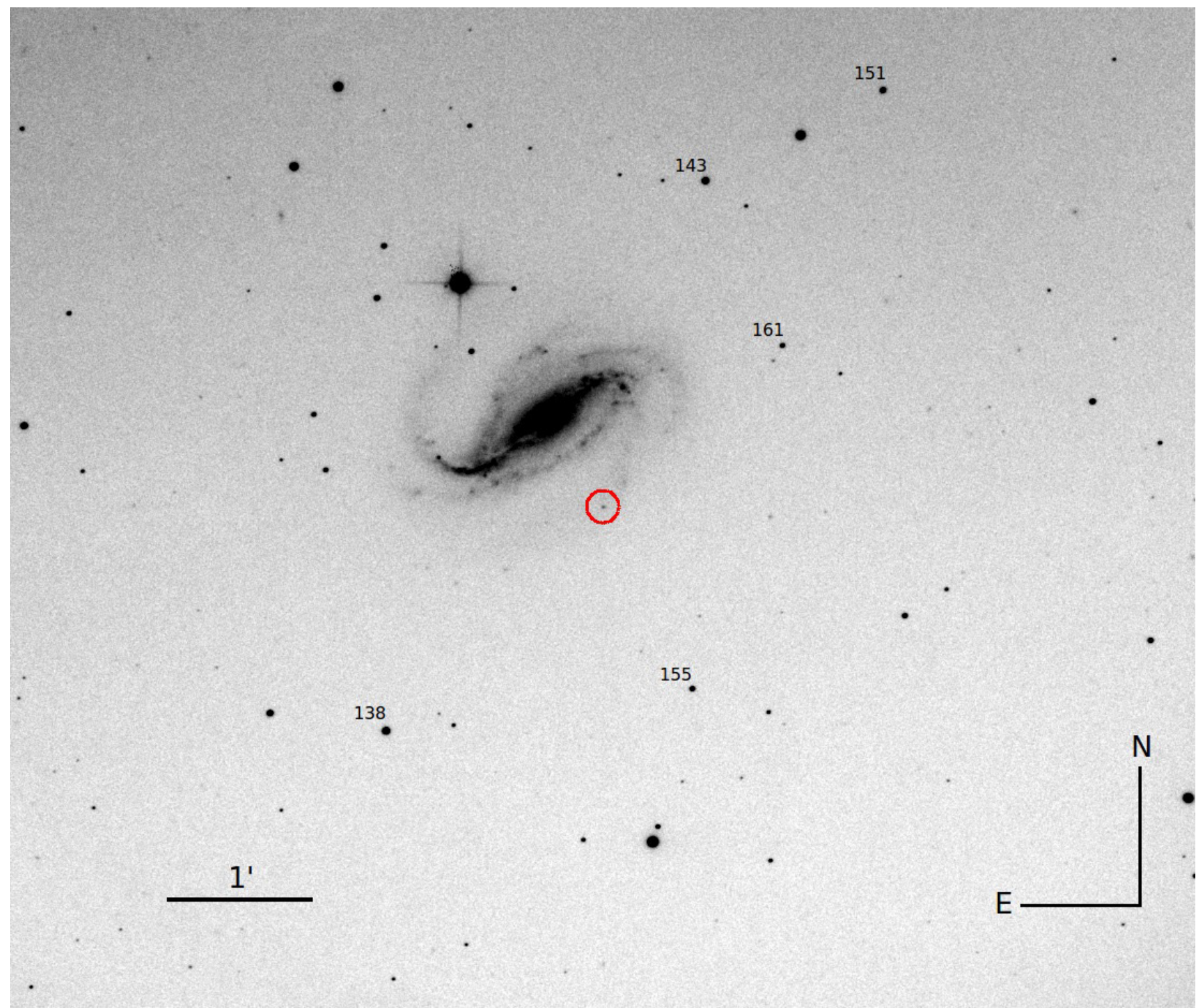

Extended Data Figure 1 | Image of SN 2016gkg in NGC 613. The image is a combination of the final 21-image series obtained at discovery. We show only the relevant region, containing the supernova (red circle), its host and the comparison stars for photometry (indicated with numbers on the upper left of each star; see Extended Data Table 1). Image obtained by V.B. 

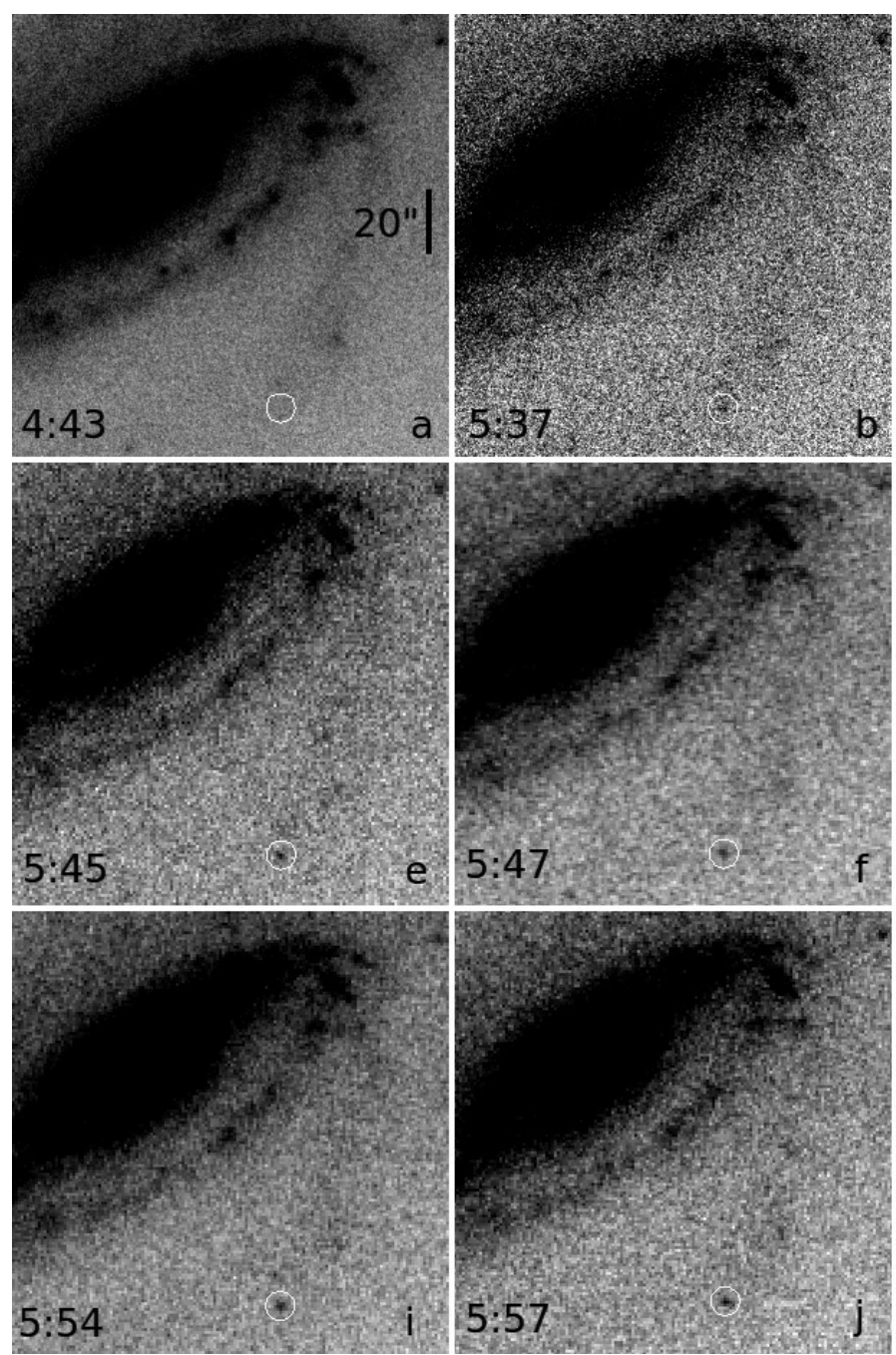

Extended Data Figure 2 | Series of discovery images of SN 2016gkg. The supernova location is indicated in all panels with a white circle.

North is up and east is to the left. The bar in a indicates a scale of $20^{\prime \prime}$. a, A combination of 40 exposures obtained before the detection of the supernova. $\mathbf{b}-\mathbf{l}$, Sequence of images obtained during the initial rise as
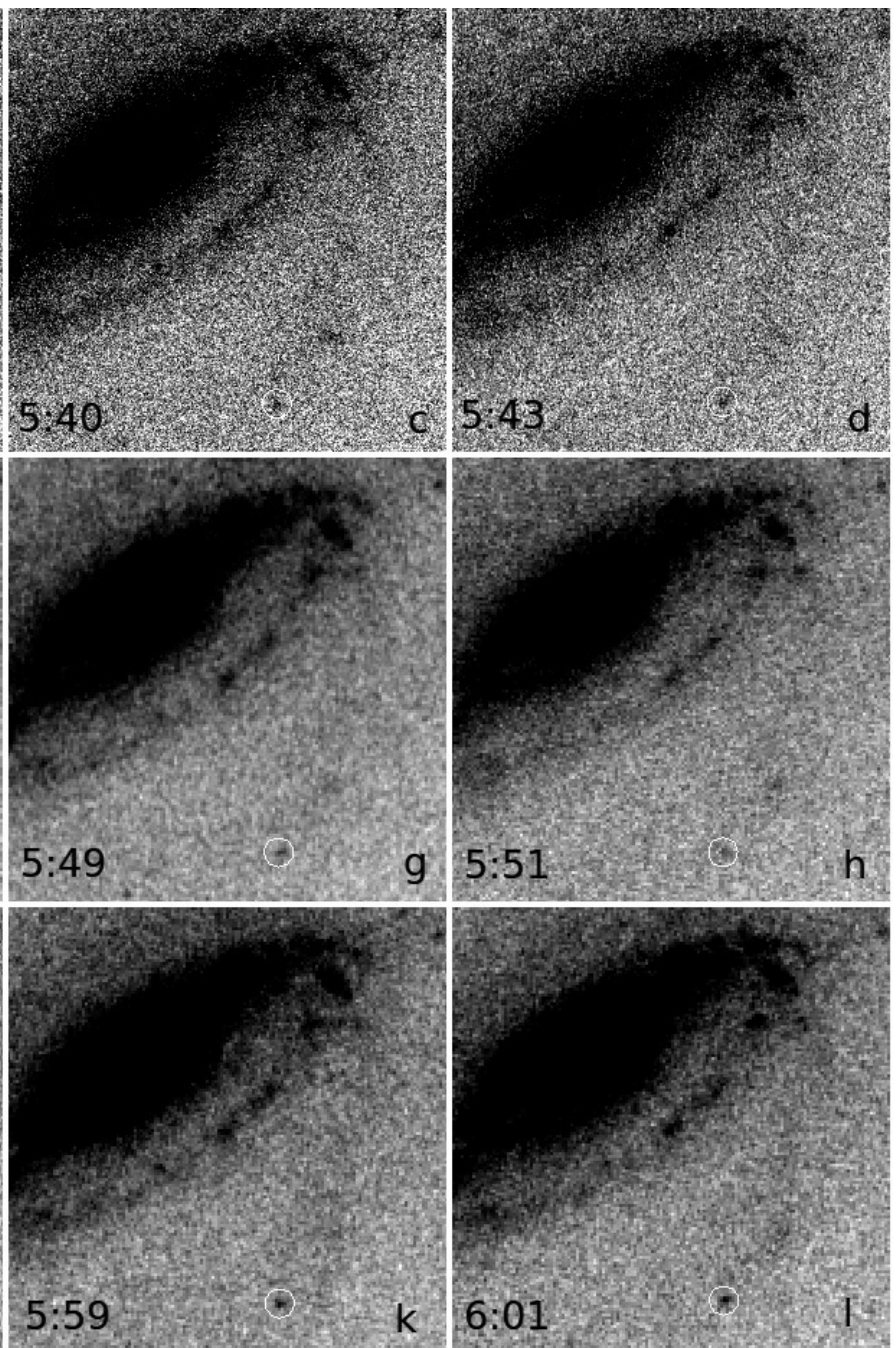

combinations of five or six individual exposures. Labels on the lower left of each panel indicate the mean UT time of the images. Photometry from the latter set of images is shown with blue diamonds in Fig. 1. Images obtained by V.B. 


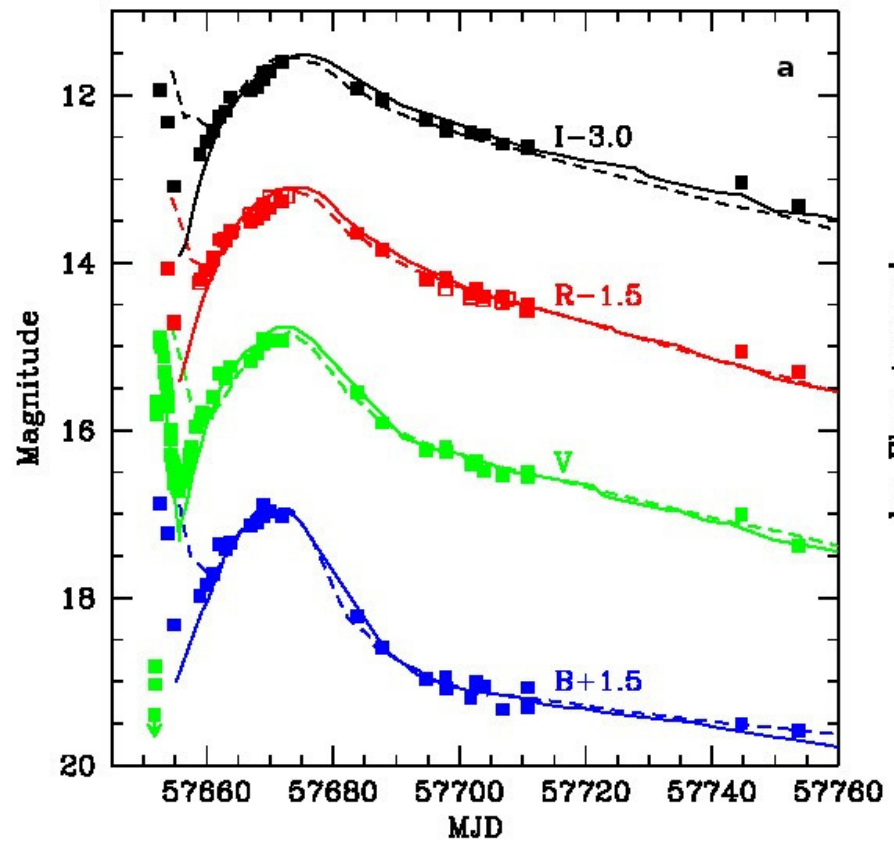

Extended Data Figure 3 | Follow-up observations of SN 2016gkg compared with those of other type IIb supernovae. a, $B V R I$ light curves for SN 2016gkg (symbols) obtained with KAIT and the Nickel telescope. $V$-band data from V.B. and J.L.S. converted from the clear band and data from Atlas, ASAS, Swift and LCOGT ${ }^{17}$ are also included. Open symbols

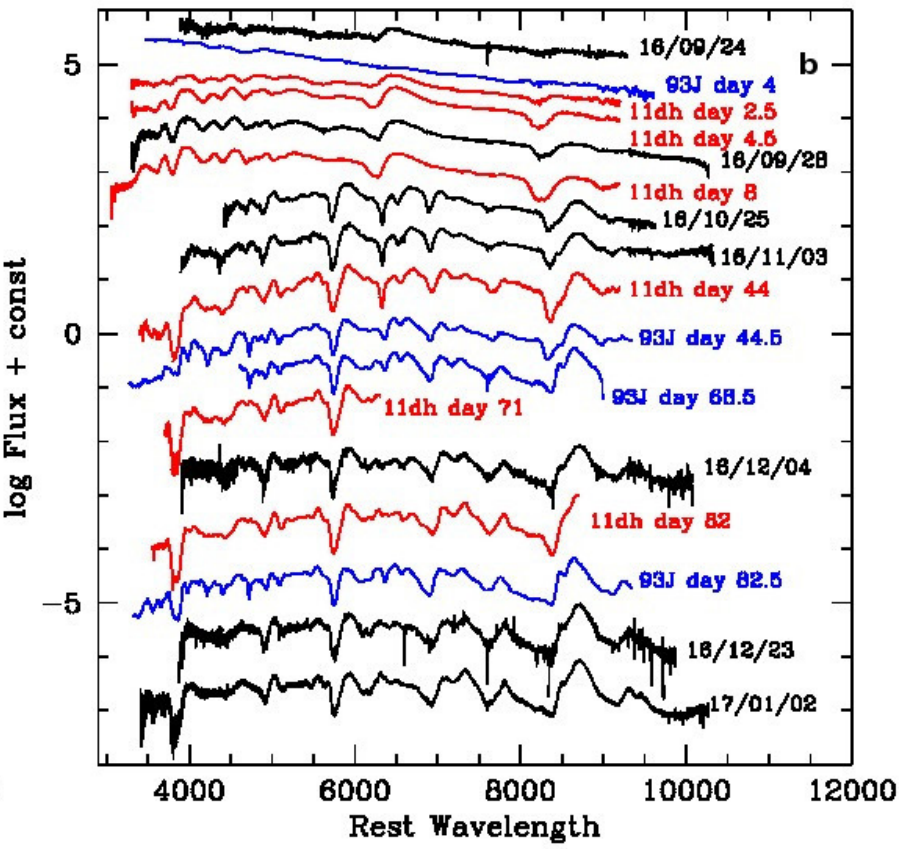

are unfiltered data from KAIT, transformed to the $R$ band. Data of type IIb supernovae SN 1993J (dashed lines) ${ }^{70}$ and SN 2011dh (solid lines) (34,38,71 $^{3}$ are included for comparison. MJD, modified Julian date. b, Optical spectra of SN $2016 \mathrm{gkg}$ (black) compared with data of the type IIb supernovae SN 1993J (blue) ${ }^{36,37}$ and SN 2011dh (red) ${ }^{38}$ at similar epochs. 

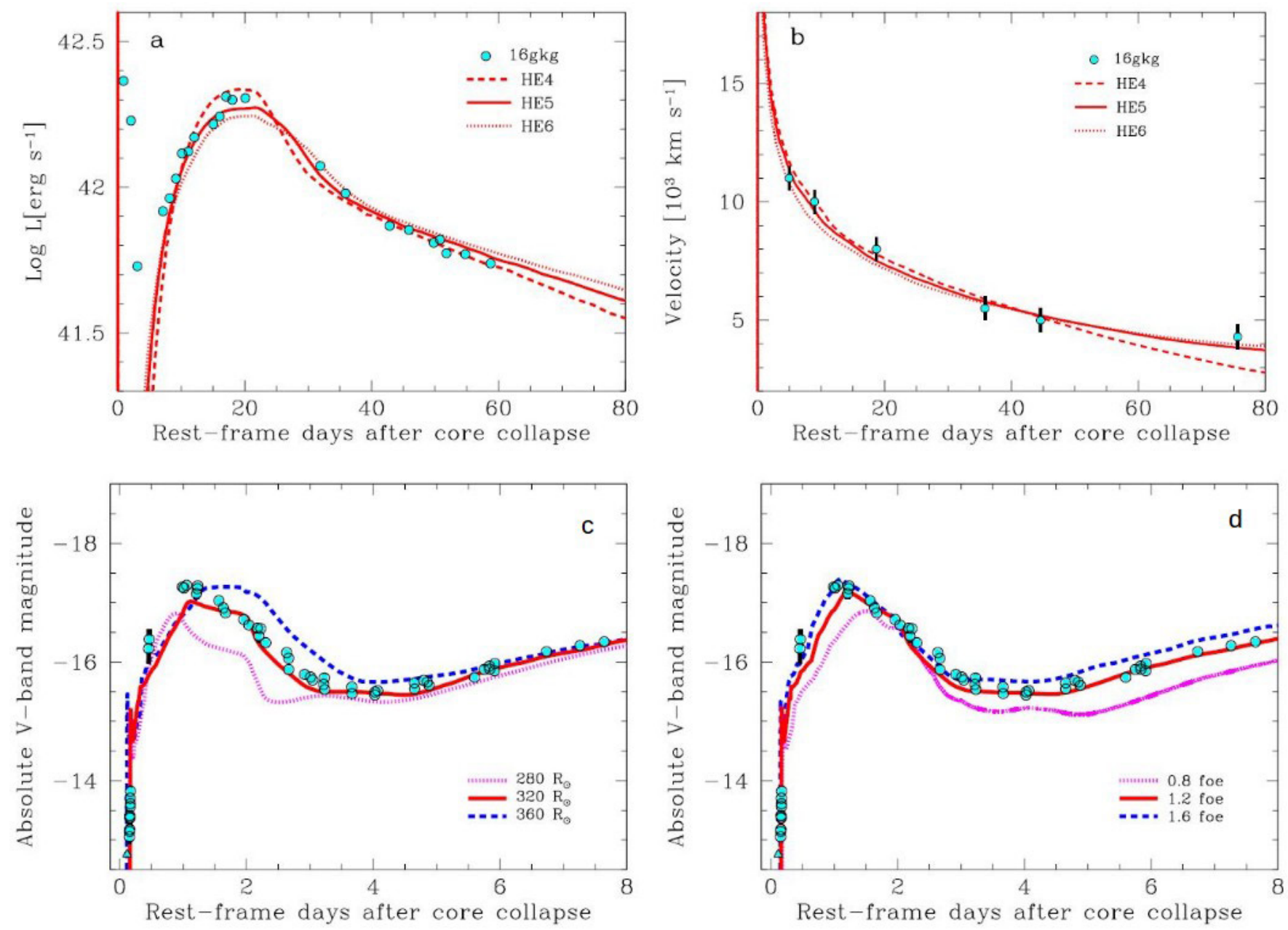

Extended Data Figure 4 | Hydrodynamical modelling of SN $2016 \mathrm{gkg}$. a, b, Model (lines) bolometric light curve (a) and photospheric velocity evolution (b) compared with observations (points) during the ${ }^{56} \mathrm{Ni}$ dominated phase. No attempt was made to reproduce the initial lightcurve decline (before day 4). c, d, Absolute $V$-band light-curve models

(lines) compared with observations (points) during the SBO and postshock cooling phases, for different progenitor radii $\left(R_{\odot} ; \mathbf{c}\right)$ and explosion energies (foe; d). Error bars are $1 \sigma$ and are shown only when they are larger than the data points. 


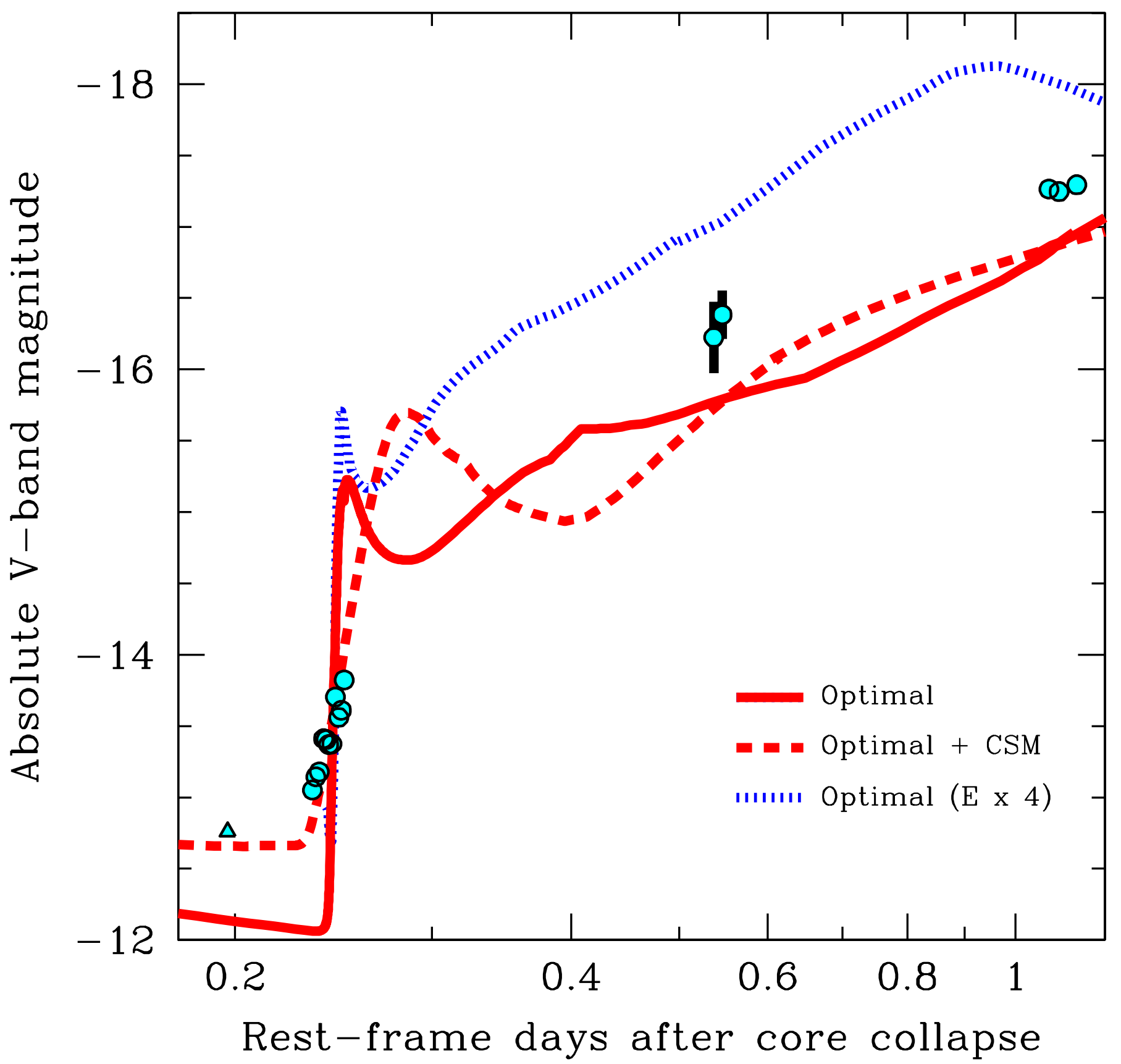

Extended Data Figure 5 | Modelling of the initial rise of SN 2016gkg. Absolute $V$-band magnitude of our preferred model (solid line), a similar slope during the SBO phase, making it even more compatible with the model including some CSM (dashed line) and a model with approximately four times larger explosion energy (dotted line), compared with the earlytime observations (points). The CSM is not necessarily in hydrostatic and thermal equilibrium. The presence of the CSM material reduces the observations, without affecting the evolution at times later than about 1 day. Even assuming an extreme explosion energy, the resulting coolingpeak slope is substantially smaller than that during the SBO. Error bars are $1 \sigma$ and are shown only when they are larger than the data points. 

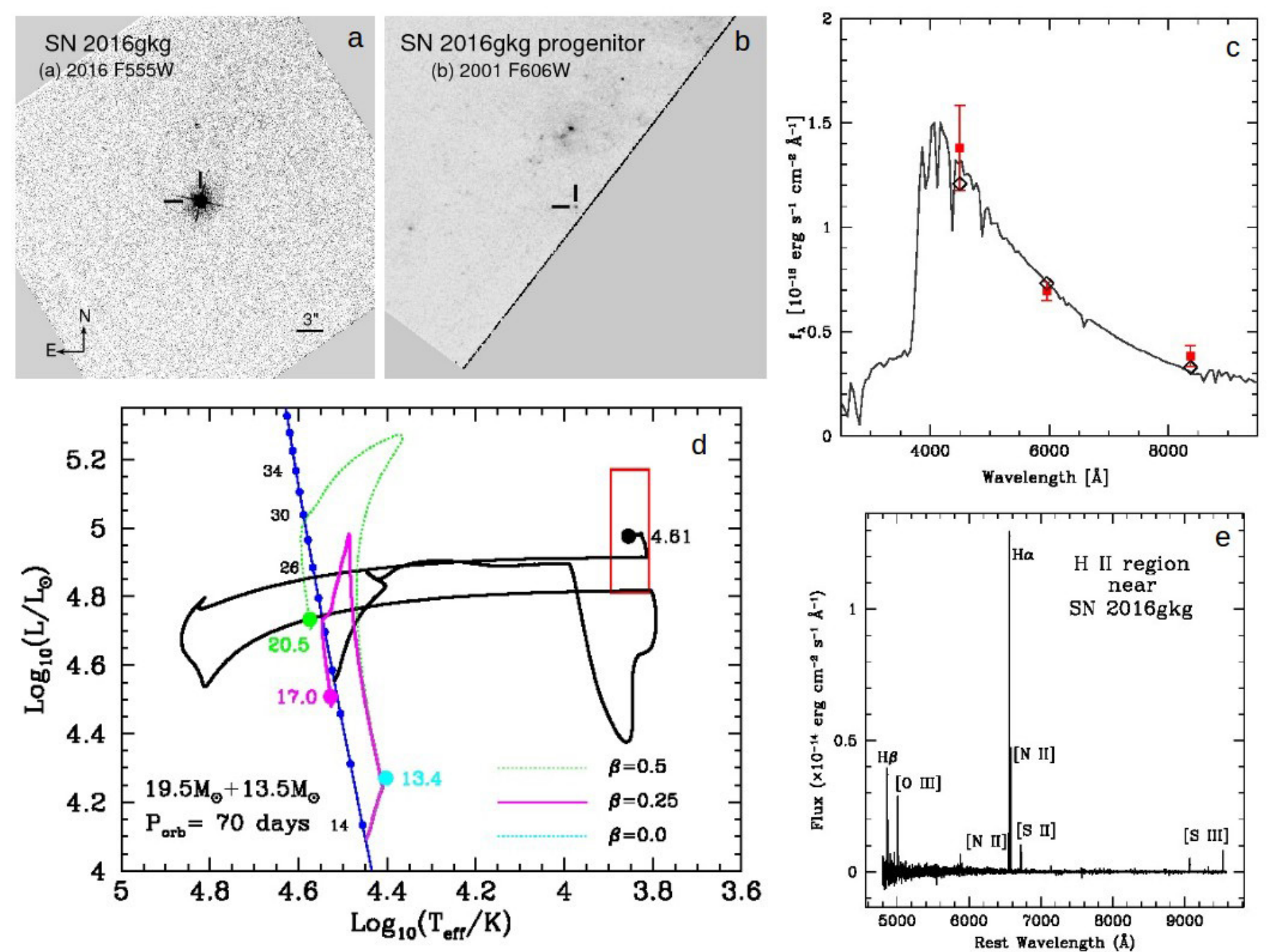

Extended Data Figure 6 The progenitor candidate and environment of SN 2016gkg. a, The HST WFC3/UVIS F555W image mosaic from 2016 October 10. b, A portion of the HST WFPC2 F606W image mosaic from 2001 August 21. The candidate position of the progenitor is indicated by tick marks. c, Stellar-atmosphere SED fit (line) to the candidate HST photometry (red squares). An H in region of which we obtained a Keck DEIMOS spectrum is seen about 8.6" north of the progenitor. Error bars are $1 \sigma . \mathbf{d}$, Evolutionary tracks on the HRD of our progenitor binary model (primary star in black; secondary star in cyan, magenta and green for different accretion efficiencies). Large circles indicate the end points of both stars, with final masses labelled, the red square shows the progenitor candidate location and the blue line is the zero-age main sequence with masses indicated. e, Spectrum of a bright $\mathrm{H}$ II region seen in $\mathbf{b}, 8.6^{\prime \prime}$ north of SN 2016gkg. 


\section{RESEARCH LETTER}

Extended Data Table 1 | Comparison stars in the field of SN 2016gkg

\begin{tabular}{|c|c|c|c|c|c|c|}
\hline Star & $\begin{array}{c}\alpha(\mathrm{J} 2000.0) \\
{[\mathrm{hh:mm:5s}]}\end{array}$ & $\begin{array}{c}\delta(\mathrm{J} 2000.0) \\
{\left[{ }^{\circ} \mathrm{I}^{\prime \prime} \mathrm{I}^{\prime}\right]}\end{array}$ & $B$ & $V$ & $R$ & $I$ \\
\hline 138 & $01: 34: 29.09$ & $-29: 29: 40.3$ & $14.741 \pm 0.038$ & $13.828 \pm 0.012$ & $13.402 \pm 0.033$ & $13.003 \pm 0.045$ \\
\hline 143 & $01: 34: 07.46$ & $-29: 21: 37.8$ & $15.015 \pm 0.013$ & $14.293 \pm 0.006$ & $13.886 \pm 0.103$ & $13.504 \pm 0.146$ \\
\hline 151 & $01: 33: 55.52$ & $-29: 20: 18.1$ & $16.091 \pm 0.081$ & $15.110 \pm 0.045$ & $14.722 \pm 0.080$ & $14.357 \pm 0.104$ \\
\hline 155 & $01: 34: 08.51$ & $-29: 29: 04.4$ & $16.376 \pm 0.110$ & $15.464 \pm 0.030$ & $15.001 \pm 0.034$ & $14.568 \pm 0.038$ \\
\hline 161 & $01: 34: 02.36$ & $-29: 24: 03.3$ & $16.694 \pm 0.056$ & $16.050 \pm 0.017$ & $\ldots$ & $\ldots$ \\
\hline
\end{tabular}


Extended Data Table 2 | Discovery imaging description and photometry of SN 2016gkg

\begin{tabular}{|c|c|c|c|c|c|c|c|c|c|c|}
\hline $\begin{array}{l}\text { Image } \\
\text { number }\end{array}$ & $\begin{array}{c}\text { JD - } \\
2457651.0 \\
\text { [day] }\end{array}$ & $\begin{array}{c}\text { Exp. } \\
\text { time } \\
{[s]}\end{array}$ & V [mag] & FWHM ["] & $\begin{array}{l}\text { zero point } \\
\text { [mag] }\end{array}$ & $\begin{array}{l}\text { Image } \\
\text { number }\end{array}$ & $\begin{array}{c}\text { JD - } \\
2457651.0 \\
\text { [day] }\end{array}$ & V [mag] & FWHM ["] & $\begin{array}{l}\text { zero point } \\
\text { [mag] }\end{array}$ \\
\hline $1-40$ & 0.69693184 & 800 & $>19.4$ & $1.93 \pm 0.13$ & $1.023 \pm 0.011$ & 74 & 0.74384259 & $19.01 \pm 0.20$ & $2.20 \pm 0.11$ & $2.925 \pm 0.008$ \\
\hline $41-57$ & 0.73615480 & 340 & $19.03 \pm 0.08$ & $1.61 \pm 0.09$ & $1.235 \pm 0.018$ & 75 & 0.74414352 & $19.04 \pm 0.58$ & $2.18 \pm 0.18$ & $2.954 \pm 0.013$ \\
\hline $41-46$ & 0.73452949 & 120 & $19.11 \pm 0.13$ & $1.59 \pm 0.10$ & $1.219 \pm 0.013$ & 76 & 0.74446759 & $18.63 \pm 0.30$ & $2.82 \pm 0.44$ & $3.071 \pm 0.020$ \\
\hline $47-52$ & 0.73629636 & 120 & $19.02 \pm 0.13$ & $1.67 \pm 0.15$ & $1.220 \pm 0.018$ & 77 & 0.74476852 & $18.58 \pm 0.20$ & $2.10 \pm 0.15$ & $2.749 \pm 0.010$ \\
\hline $53-57$ & 0.73793518 & 100 & $18.98 \pm 0.12$ & $1.57 \pm 0.12$ & $1.222 \pm 0.020$ & $78-98$ & 0.74817347 & $18.48 \pm 0.05$ & $2.06 \pm 0.07$ & $2.780 \pm 0.016$ \\
\hline 41 & 0.73376157 & 20 & $18.95 \pm 0.39$ & $1.71 \pm 0.20$ & $1.244 \pm 0.023$ & $78-83$ & 0.74595499 & $18.46 \pm 0.08$ & $2.28 \pm 0.08$ & $2.845 \pm 0.012$ \\
\hline 42 & 0.73410880 & 20 & $19.55 \pm 0.36$ & $2.21 \pm 0.24$ & $1.332 \pm 0.021$ & $84-88$ & 0.74763662 & $18.60 \pm 0.09$ & $2.00 \pm 0.08$ & $2.814 \pm 0.025$ \\
\hline 43 & 0.73439815 & 20 & $18.95 \pm 0.19$ & $1.53 \pm 0.35$ & $1.248 \pm 0.015$ & $89-93$ & 0.74904883 & $18.55 \pm 0.09$ & $2.00 \pm 0.06$ & $2.780 \pm 0.014$ \\
\hline 44 & 0.73468750 & 20 & $18.77 \pm 0.26$ & $1.53 \pm 0.21$ & $1.220 \pm 0.010$ & $94-98$ & 0.75049746 & $18.34 \pm 0.08$ & $2.00 \pm 0.07$ & $2.783 \pm 0.018$ \\
\hline 45 & 0.73496528 & 20 & $19.65 \pm 0.38$ & $1.67 \pm 0.38$ & $1.199 \pm 0.013$ & 78 & 0.74512731 & $18.70 \pm 0.19$ & $2.06 \pm 0.10$ & $2.708 \pm 0.012$ \\
\hline 46 & 0.73525463 & 20 & $19.41 \pm 0.42$ & $1.56 \pm 0.28$ & $1.238 \pm 0.016$ & 79 & 0.74550926 & $18.39 \pm 0.14$ & $2.48 \pm 0.28$ & $2.866 \pm 0.016$ \\
\hline 47 & 0.73554398 & 20 & $19.01 \pm 0.31$ & $2.13 \pm 0.46$ & $1.165 \pm 0.009$ & 80 & 0.74582176 & $18.80 \pm 0.31$ & $2.35 \pm 0.14$ & $2.953 \pm 0.011$ \\
\hline 48 & 0.73585648 & 20 & $19.26 \pm 0.41$ & $1.53 \pm 0.52$ & $1.221 \pm 0.020$ & 81 & 0.74612269 & $18.76 \pm 0.35$ & $2.89 \pm 0.44$ & $3.103 \pm 0.008$ \\
\hline 49 & 0.73614583 & 20 & $19.14 \pm 0.33$ & $1.71 \pm 0.29$ & $1.201 \pm 0.015$ & 82 & 0.74642361 & $18.11 \pm 0.15$ & $2.22 \pm 0.10$ & $2.770 \pm 0.009$ \\
\hline 50 & 0.73644676 & 20 & $18.45 \pm 0.12$ & $2.07 \pm 0.27$ & $1.274 \pm 0.022$ & 83 & 0.74672454 & $18.47 \pm 0.17$ & $2.22 \pm 0.15$ & $2.741 \pm 0.026$ \\
\hline 51 & 0.73673611 & 20 & $19.40 \pm 0.39$ & $1.84 \pm 0.43$ & $1.208 \pm 0.023$ & 84 & 0.74706019 & $18.43 \pm 0.18$ & $2.12 \pm 0.12$ & $2.770 \pm 0.026$ \\
\hline 52 & 0.73704861 & 20 & $19.59 \pm 0.50$ & $1.64 \pm 0.55$ & $1.174 \pm 0.014$ & 85 & 0.74737269 & $18.67 \pm 0.16$ & $1.97 \pm 0.09$ & $2.756 \pm 0.014$ \\
\hline 53 & 0.73734954 & 20 & $19.17 \pm 0.24$ & $1.52 \pm 0.27$ & $1.166 \pm 0.015$ & 86 & 0.74765046 & $18.84 \pm 0.31$ & $1.97 \pm 0.15$ & $2.769 \pm 0.017$ \\
\hline 54 & 0.73765046 & 20 & $19.23 \pm 0.30$ & $1.77 \pm 0.23$ & $1.255 \pm 0.014$ & 87 & 0.74791667 & $18.55 \pm 0.15$ & $2.81 \pm 0.30$ & $2.979 \pm 0.025$ \\
\hline 55 & 0.73793982 & 20 & $18.67 \pm 0.20$ & $1.63 \pm 0.51$ & $1.197 \pm 0.013$ & 88 & 0.74818287 & $18.43 \pm 0.15$ & $1.78 \pm 0.16$ & $2.753 \pm 0.017$ \\
\hline 56 & 0.73822917 & 20 & $19.02 \pm 0.29$ & $1.58 \pm 0.42$ & $1.218 \pm 0.024$ & 89 & 0.74843750 & $18.63 \pm 0.16$ & $2.01 \pm 0.09$ & $2.777 \pm 0.014$ \\
\hline 57 & 0.73850694 & 20 & $18.90 \pm 0.25$ & $2.05 \pm 0.30$ & $1.267 \pm 0.019$ & 90 & 0.74870370 & $18.35 \pm 0.14$ & $1.90 \pm 0.10$ & $2.756 \pm 0.018$ \\
\hline $58-77$ & 0.74201745 & 400 & $18.82 \pm 0.07$ & $2.03 \pm 0.10$ & $2.787 \pm 0.011$ & 91 & 0.74906250 & $18.62 \pm 0.17$ & $2.38 \pm 1.28$ & $2.659 \pm 0.010$ \\
\hline $58-62$ & 0.73999995 & 100 & $18.75 \pm 0.12$ & $1.85 \pm 0.12$ & $2.822 \pm 0.014$ & 92 & 0.74938657 & $18.19 \pm 0.13$ & $2.17 \pm 0.11$ & $2.817 \pm 0.012$ \\
\hline $63-67$ & 0.74130100 & 100 & $18.75 \pm 0.08$ & $2.12 \pm 0.09$ & $2.825 \pm 0.013$ & 93 & 0.74965278 & $19.17 \pm 0.31$ & $2.14 \pm 1.17$ & $2.684 \pm 0.022$ \\
\hline $68-72$ & 0.74262035 & 100 & $18.79 \pm 0.10$ & $2.18 \pm 0.11$ & $2.800 \pm 0.011$ & 94 & 0.74994213 & $18.31 \pm 0.14$ & $2.10 \pm 0.11$ & $2.726 \pm 0.014$ \\
\hline $73-77$ & 0.74414861 & 100 & $18.79 \pm 0.15$ & $2.09 \pm 0.12$ & $2.826 \pm 0.012$ & 95 & 0.75023148 & $18.43 \pm 0.18$ & $2.61 \pm 0.15$ & $2.876 \pm 0.023$ \\
\hline 58 & 0.73947917 & 20 & $18.59 \pm 0.26$ & $1.66 \pm 0.14$ & $2.762 \pm 0.016$ & 96 & 0.75050926 & $18.23 \pm 0.14$ & $1.92 \pm 0.15$ & $2.707 \pm 0.021$ \\
\hline 59 & 0.73974537 & 20 & $18.93 \pm 0.21$ & $2.07 \pm 0.11$ & $2.785 \pm 0.016$ & 97 & 0.75077546 & $18.51 \pm 0.15$ & $2.03 \pm 0.14$ & $2.822 \pm 0.020$ \\
\hline 60 & 0.74000000 & 20 & $18.82 \pm 0.29$ & $1.91 \pm 0.12$ & $2.827 \pm 0.016$ & 98 & 0.75103009 & $18.32 \pm 0.14$ & $1.83 \pm 0.17$ & $2.722 \pm 0.023$ \\
\hline 61 & 0.74025463 & 20 & $19.20 \pm 0.28$ & $1.95 \pm 0.16$ & $2.803 \pm 0.014$ & & & & & \\
\hline 62 & 0.74052083 & 20 & $18.53 \pm 0.20$ & $1.79 \pm 0.15$ & $2.786 \pm 0.013$ & & & & & \\
\hline 63 & 0.74077546 & 20 & $19.05 \pm 0.29$ & $2.54 \pm 0.11$ & $2.926 \pm 0.009$ & & & & & \\
\hline 64 & 0.74104167 & 20 & $18.35 \pm 0.16$ & $2.43 \pm 0.15$ & $2.929 \pm 0.028$ & & & & & \\
\hline 65 & 0.74129630 & 20 & $19.01 \pm 0.23$ & $2.01 \pm 0.10$ & $2.777 \pm 0.019$ & & & & & \\
\hline 66 & 0.74156250 & 20 & $18.52 \pm 0.15$ & $2.05 \pm 0.07$ & $2.772 \pm 0.013$ & & & & & \\
\hline 67 & 0.74182870 & 20 & $19.04 \pm 0.29$ & $1.93 \pm 0.12$ & $2.789 \pm 0.011$ & & & & & \\
\hline 68 & 0.74208333 & 20 & $18.46 \pm 0.16$ & $2.32 \pm 0.08$ & $2.870 \pm 0.022$ & & & & & \\
\hline 69 & 0.74234954 & 20 & $19.88 \pm 0.49$ & $2.38 \pm 0.27$ & $2.888 \pm 0.012$ & & & & & \\
\hline 70 & 0.74261574 & 20 & $19.32 \pm 0.35$ & $2.08 \pm 0.11$ & $2.807 \pm 0.012$ & & & & & \\
\hline 71 & 0.74287037 & 20 & $18.71 \pm 0.21$ & $1.85 \pm 0.13$ & $2.746 \pm 0.020$ & & & & & \\
\hline 72 & 0.74318287 & 20 & $18.83 \pm 0.22$ & $2.41 \pm 0.07$ & $2.825 \pm 0.019$ & & & & & \\
\hline 73 & 0.74351852 & 20 & $18.94 \pm 0.27$ & $1.82 \pm 0.17$ & $2.632 \pm 0.018$ & & & & & \\
\hline
\end{tabular}




\section{RESEARCH LETTER}

Extended Data Table 3 | Follow-up BVRI and clear photometry of SN 2016gkg

\begin{tabular}{|c|c|c|c|c|c|c|}
\hline MJD & $B$ & V & $R$ & $I$ & Clear & Source \\
\hline 57652.1 & $15.37 \pm 0.35$ & $14.94 \pm 0.05$ & $\ldots$ & $14.93 \pm 0.06$ & $\ldots$ & Buso \\
\hline 57652.3 & $\ldots$ & $14.95 \pm 0.03$ & $\ldots$ & $\ldots$ & $\ldots$ & Sánchez \\
\hline 57653.3 & $15.73 \pm 0.14$ & $15.60 \pm 0.08$ & $15.57 \pm 0.06$ & $15.32 \pm 0.10$ & $\ldots$ & KAIT \\
\hline 57654.3 & $16.82 \pm 0.16$ & $16.43 \pm 0.12$ & $16.23 \pm 0.08$ & $16.09 \pm 0.12$ & $16.22 \pm 0.03$ & KAIT \\
\hline 57658.4 & $16.47 \pm 0.08$ & $15.89 \pm 0.04$ & $15.70 \pm 0.04$ & $15.70 \pm 0.06$ & $15.74 \pm 0.04$ & KAIT \\
\hline 57659.4 & $16.34 \pm 0.08$ & $15.80 \pm 0.04$ & $15.58 \pm 0.04$ & $15.56 \pm 0.06$ & $15.62 \pm 0.04$ & KAIT \\
\hline 57660.4 & $16.21 \pm 0.08$ & $15.60 \pm 0.04$ & $15.43 \pm 0.02$ & $15.42 \pm 0.04$ & $15.45 \pm 0.05$ & KAIT \\
\hline 57661.4 & $15.86 \pm 0.04$ & $15.33 \pm 0.02$ & $15.22 \pm 0.02$ & $15.26 \pm 0.04$ & $\ldots$ & Nickel \\
\hline 57662.4 & $15.92 \pm 0.12$ & $15.38 \pm 0.06$ & $15.22 \pm 0.04$ & $15.19 \pm 0.06$ & $15.22 \pm 0.03$ & KAIT \\
\hline 57663.3 & $15.84 \pm 0.10$ & $15.24 \pm 0.06$ & $15.14 \pm 0.04$ & $15.02 \pm 0.06$ & $15.12 \pm 0.05$ & KAIT \\
\hline 57666.4 & $15.63 \pm 0.08$ & $15.17 \pm 0.04$ & $15.01 \pm 0.02$ & $14.94 \pm 0.04$ & $14.93 \pm 0.02$ & KAIT \\
\hline 57667.4 & $15.59 \pm 0.06$ & $15.07 \pm 0.04$ & $14.98 \pm 0.02$ & $14.90 \pm 0.04$ & $14.93 \pm 0.02$ & KAIT \\
\hline 57668.4 & $15.39 \pm 0.02$ & $14.91 \pm 0.02$ & $14.80 \pm 0.02$ & $14.74 \pm 0.02$ & $\cdots$ & Nickel \\
\hline 57668.4 & $15.53 \pm 0.16$ & $14.96 \pm 0.12$ & $14.92 \pm 0.08$ & $14.81 \pm 0.10$ & $14.82 \pm 0.06$ & KAIT \\
\hline 57669.4 & $15.46 \pm 0.10$ & $14.93 \pm 0.06$ & $14.85 \pm 0.04$ & $14.72 \pm 0.08$ & $14.72 \pm 0.04$ & KAIT \\
\hline 57671.4 & $15.52 \pm 0.10$ & $14.93 \pm 0.10$ & $14.77 \pm 0.06$ & $14.61 \pm 0.10$ & $14.70 \pm 0.05$ & KAIT \\
\hline 57672.3 & $\ldots$ & $\ldots$ & $\ldots$ & $\cdots$ & $14.71 \pm 0.04$ & KAIT \\
\hline 57683.3 & $16.72 \pm 0.02$ & $15.55 \pm 0.02$ & $15.15 \pm 0.02$ & $14.92 \pm 0.02$ & $\ldots$ & Nickel \\
\hline 57687.3 & $17.10 \pm 0.04$ & $15.91 \pm 0.02$ & $15.35 \pm 0.02$ & $15.05 \pm 0.02$ & $\ldots$ & Nickel \\
\hline 57694.3 & $17.46 \pm 0.16$ & $16.24 \pm 0.06$ & $15.71 \pm 0.04$ & $15.29 \pm 0.04$ & $15.70 \pm 0.02$ & KAIT \\
\hline 57697.3 & $17.44 \pm 0.04$ & $16.20 \pm 0.02$ & $15.68 \pm 0.02$ & $15.36 \pm 0.02$ & $\ldots$ & Nickel \\
\hline 57697.4 & $17.58 \pm 0.18$ & $16.26 \pm 0.08$ & $15.73 \pm 0.04$ & $15.42 \pm 0.06$ & $15.81 \pm 0.04$ & KAIT \\
\hline 57701.3 & $17.69 \pm 0.18$ & $16.40 \pm 0.06$ & $15.87 \pm 0.04$ & $15.44 \pm 0.04$ & $15.92 \pm 0.05$ & KAIT \\
\hline 57702.3 & $17.51 \pm 0.06$ & $16.36 \pm 0.02$ & $15.81 \pm 0.02$ & $\cdots$ & $\ldots$ & Nickel \\
\hline 57703.3 & $17.55 \pm 0.36$ & $16.49 \pm 0.14$ & $15.91 \pm 0.08$ & $15.47 \pm 0.08$ & $15.92 \pm 0.04$ & KAIT \\
\hline 57706.3 & $17.83 \pm 0.46$ & $16.53 \pm 0.14$ & $15.91 \pm 0.08$ & $15.58 \pm 0.08$ & $15.97 \pm 0.05$ & KAIT \\
\hline 57707.2 & $\cdots$ & $\cdots$ & $\cdots$ & $\cdots$ & $15.93 \pm 0.07$ & KAIT \\
\hline 57710.3 & $17.80 \pm 0.20$ & $16.55 \pm 0.06$ & $16.06 \pm 0.04$ & $15.62 \pm 0.04$ & $16.07 \pm 0.06$ & KAIT \\
\hline 57710.3 & $17.57 \pm 0.12$ & $16.50 \pm 0.04$ & $16.00 \pm 0.02$ & $15.60 \pm 0.04$ & $\cdots$ & Nickel \\
\hline 57744.2 & $18.00 \pm 0.04$ & $17.00 \pm 0.02$ & $16.56 \pm 0.02$ & $16.04 \pm 0.02$ & $\ldots$ & Nickel \\
\hline 57753.1 & $18.09 \pm 0.04$ & $17.38 \pm 0.06$ & $16.80 \pm 0.02$ & $16.32 \pm 0.02$ & $\ldots$ & Nickel \\
\hline
\end{tabular}


Extended Data Table 4 | $\mathrm{H}$ II region line fluxes

\begin{tabular}{|c|c|}
\hline Line & $\begin{array}{c}\text { Flux } \\
{\left[10-15 \mathrm{erg} \mathrm{cm}^{-2} \mathrm{~s}^{-1}\right]}\end{array}$ \\
\hline $\mathrm{H} \beta$ & $19.2 \pm 0.4$ \\
\hline$[\mathrm{O} \mathrm{III]} \lambda 4959$ & $2.71 \pm 0.31$ \\
\hline$[\mathrm{O} \mathrm{III]} \lambda 5007$ & $9.64 \pm 0.29$ \\
\hline$[\mathrm{N} \mathrm{II}] \lambda 6548$ & $5.74 \pm 0.05$ \\
\hline $\mathrm{H} \alpha$ & $55.2 \pm 0.3$ \\
\hline$[\mathrm{N} \mathrm{II}] \lambda 6584$ & $19.4 \pm 0.1$ \\
\hline$[\mathrm{S} \mathrm{II}] \lambda 6717$ & $4.06 \pm 0.05$ \\
\hline$[\mathrm{S} \mathrm{II}] \lambda 6731$ & $2.75 \pm 0.07$ \\
\hline$[\mathrm{S} \mathrm{III]} \lambda 9069$ & $1.67 \pm 0.02$ \\
\hline$[\mathrm{S} \mathrm{III]} \lambda 9532$ & $2.62 \pm 0.03$ \\
\hline
\end{tabular}

